\title{
Stabilized FE Simulation of Prototype Thermal-Hydraulics Problems with Integrated Adjoint-based Capabilities
}

\author{
J.N. Shadid ${ }^{a, d, *}$, T.M. Smith ${ }^{\text {b }}$, E.C. Cyr ${ }^{\mathrm{c}}$, T.M. Wildey ${ }^{\mathrm{c}}$, R.P. Pawlowski ${ }^{\mathrm{b}}$ \\ ${ }^{a}$ Sandia National Laboratories, Computational Mathematics Department \\ ${ }^{b}$ Sandia National Laboratories, Multiphysics Applications Department \\ ${ }^{c}$ Sandia National Laboratories, Optimization and UQ Department \\ ${ }^{d}$ Department of Mathematics and Statistics, University of New Mexico
}

\begin{abstract}
A critical aspect of applying modern computational solution methods to complex multiphysics systems of relevance to nuclear reactor modeling, is the assessment of the predictive capability of specific proposed mathematical models. In this respect the understanding of numerical error, the sensitivity of the solution to parameters associated with input data, boundary condition uncertainty, and mathematical models is critical. Additionally, the ability to evaluate and or approximate the model efficiently, to allow development of a reasonable level of statistical diagnostics of the mathematical model and the physical system, is of central importance. In this study we report on initial efforts to apply integrated adjoint-based computational analysis and automatic differentiation tools to begin to address these issues. The study is carried out in the context of a Reynolds averaged Navier-Stokes approximation to turbulent fluid flow and heat transfer using a particular spatial discretization based on implicit fully-coupled stabilized FE methods. Initial results are presented that show the promise of these computational techniques in the context of nuclear reactor relevant prototype thermal-hydraulics problems.
\end{abstract}

Keywords: Reynolds Averaged Navier-Stokes, stabilized finite elements, adjoints, sensitivities, error-estimation, uncertainty quantification

\section{Introduction}

Full scale system level thermal hydraulic numerical simulations of high Reynolds number $(R e)$ flows in pressureized water reactors (PWR), with coupled neutronics and conjugate heat transfer, remain very computationally challenging problems. For this class of problems solving the time averaged Reynolds averaged Navier-Stokes (RANS) equations with a turbulence closure model is currently the only feasible option for obtaining solutions. Until unsteady-RANS, or large-eddy simulation, approaches become computationally tractable, there is a need to develop and evaluate steady-state RANS models to understand the strengths and weaknesses of the model as well as quantify uncertainties. For this reason RANS modeling capabilities are being developed in the

*Corresponding author: John N. Shadid (jnshadi@sandia.gov)

Preprint submitted to J. Comput. Phys.

March 25, 2016 
Consortium for Advanced Simulation of Light Water Reactors (CASL) program to solve thermal hydraulic challenge problems at full system scale.

The Spalart-Allmaras [40] (SARANS) turbulence model is used to provide closure for the RANS equations. This model includes a single time dependent advection-diffusionreaction transport equation for the eddy viscosity. This viscosity couples to the NavierStokes equations using the Boussinesq approximation. Therefore, the stress tensor in the momentum equation is modified by an additional "effective" viscosity. A similar closure is used for the turbulent heat flux where in this case the Fourier heat law is modified by substituting an "effective" conductivity for the molecular value.

Predictive computational analysis and uncertainty quantification (UQ) for multiphysics has many open challenges including uncertainties arising from; discretization errors, boundary and initial conditions, model parameters and physical parameters. Computational analysis and design often focus on a set of Quantities-of-Interest (QoI) or functionals of the solution rather than the entire state. The error and uncertainty propagation along with sensitivity characteristics for specific QoI can be significantly different from the PDE solution itself. We are addressing these challenges in studies of a moderate number of QoI dependent on a large-number of parameters using fully coupled NewtonKrylov and integrated adjoint-based methods to accurately and efficiently compute numerical error-estimates, to conduct sensitivity analysis and to develop gradient-enhanced surrogate models. Adjoint methods have been well studied and demonstrated for systems of PDEs (see; Bangerth\&Rannacher [3], Becker [5], Eriksson et al. [14], Estep et al. [16]). Additionally, sensitivity and uncertainty analysis has been developed for nuclear applications (see e.g., [8] and [9], [31], [19] and the references there in). In this study we consider the application and evaluation of adjoint-based methods for sensitivity analysis, the development of surrogate models for UQ analysis, and the estimation of discretization error for reasonably complex RANS models that are applicable to the simulation of CASL relevant prototype thermal-hydraulics problems.

The application of adjoint-based techniques, for the evaluation of steady-state RANS models, to complex multiphysics systems is challenging, and robust and efficient nonlinear and linear iterative solution methods are required. The robust solution method approach that we have developed is to use fully-implicit and fully-coupled nonlinear/linear iterative solvers for unstructured finite element approximations of the governing balance equations. These methods can be very efficient for achieving steady-state solutions and for coupled multiphysics simulations (e.g., turbulent flow and conjugate heat transfer). Solutions can be obtained by integrating a transient solution to steady-state with very-large time steps (pseudo-time stepping), often directly to steady-state, and also by parameter continuation where an initial simulation at a particular parameter value (e.g., $R e$ ) is then continued to trace out an entire solution branch (see e.g., [32]). This solution methodology is currently implemented in the Drekar::CFD code [30]. An important feature of the Drekar::CFD code infrastructure is that it supports error-estimation, sensitivity and UQ algorithms that can use integrated adjoint-based techniques. This type of approach can be very efficient for the class of adjoint-based, error-estimation, sensitivity analysis and UQ surrogate construction problems requiring repeated function evaluations along with computations of the gradients of the QoI with respect to the uncertain parameters of the problem.

A unique aspect of the Drekar::CFD code is the use of template-based embedded automatic differentiation (AD) technology described in [28, 29]. This is used for automated 
construction of the Jacobian operator required for the fully-coupled implicit solution approaches. This gives Drekar::CFD developers the capability to rapidly implement new models, while easily maintaining the implicit solution approach with mathematically accurate Jacobians. While useful in the context of forward simulation, we believe the AD technology is vitally important to go beyond forward simulation methodologies requiring additional sensitivities. The set of possible responses and parameters required by an analyst may represent a fairly large space of possible use cases. The AD technology combined with a flexible runtime system, like the directed acyclic graph employed in Drekar::CFD (see [26] for description), allows for responses and parameters to be specified at runtime. Without such a tool an analyst would be required to hand code sensitivities for every response/parameter pair, resulting in a substantial increase in development time and difficulty.

A detailed description of Drekar::CFD can be found in the theory manual which is available as a CASL milestone report [30]. Here, the main ideas behind the RANS models and how they couple to the Navier-Stokes equations will be presented. Two examples taken from Smith et al. [39] are shown in Figures 1(a) and 1(b) showing temperature contours in a single span of a $3 \times 3 \mathrm{rod} /$ spacer grid sub-channel prototype. In the top panel, a constant wall heat flux has been assumed. In the bottom, the conjugate problem is solved assuming constant volumetric heat source in the rods. These figures illustrate the complexity involved in solving this class of thermal hydraulic problems.

In the remaining sections, RANS equations and turbulence models appropriate for solving incompressible high Re flows with heat transfer are described along with the modifications to the momentum equations necessary for closure. Then the numerical methods are described. Verification of heat transfer is provided by solving fully developed laminar flow in a pipe and comparing the Nusselt number to analytically derived values of Kays\&Crawford [22]. Next a verification of the methodology for computing error estimate and sensitivity to QoI is presented by solving fully developed laminar rectangular duct flow for which an analytic solution exists. The problem establishes the accuracy of the error estimates and parameter sensitivities to a small group of QoI that can also be computed analytically. The results section is split between two studies; turbulent swirling flow through a sudden expansion pipe, and heat transport in fully developed turbulent pipe flow. In both cases, error estimation and parameter sensitivities to a select group of QoIs are presented. Finally, a UQ study of QoI in the case of fully developed pipe flow is presented. The final section of the paper provides some conclusions.

\section{Background and Mathematical Formulation}

\subsection{Governing Equations}

The equations governing incompressible fluid motion with heat transport are continuity, momentum and energy, in this application, written in terms of temperature. The residual form of the equations are listed in Table 1 which is appropriate for a finite element discretization. In this study, all body forces and viscous dissipation have been neglected. In these equations, $\rho$ is the density (a constant), $\overline{\mathbf{u}}=\bar{u}_{i}, i=1,2,3$ is the "Reynolds Averaged" velocity vector with index $i$ representing Cartesian components in the $(x, y, z)$ directions, $\bar{T}$ is temperature, $C_{p}$ the specific heat at constant pressure, $\mathbf{T}$ is 


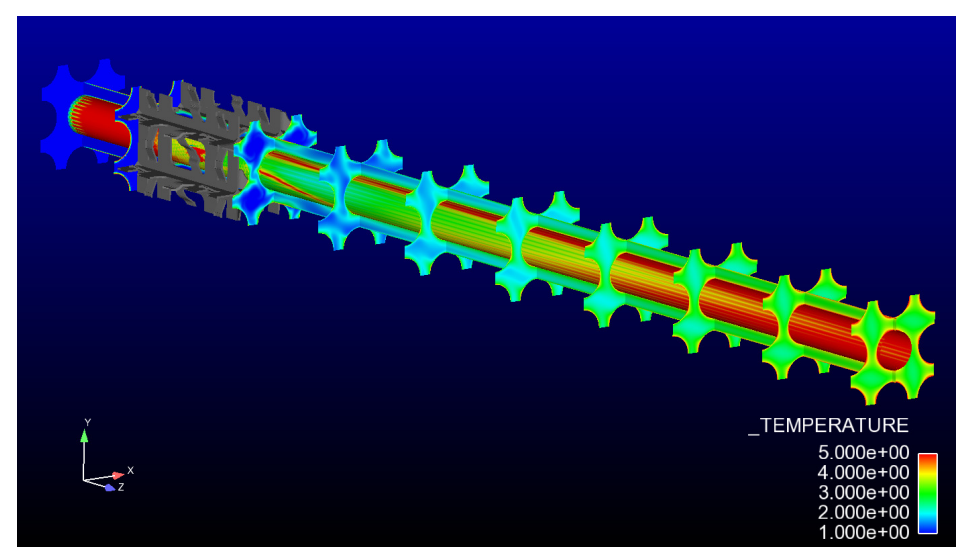

(a) Constant rod heat flux $\dot{q}_{w}^{\prime \prime}, \operatorname{Pr}=1.45,15 \mathrm{M}$ element mesh

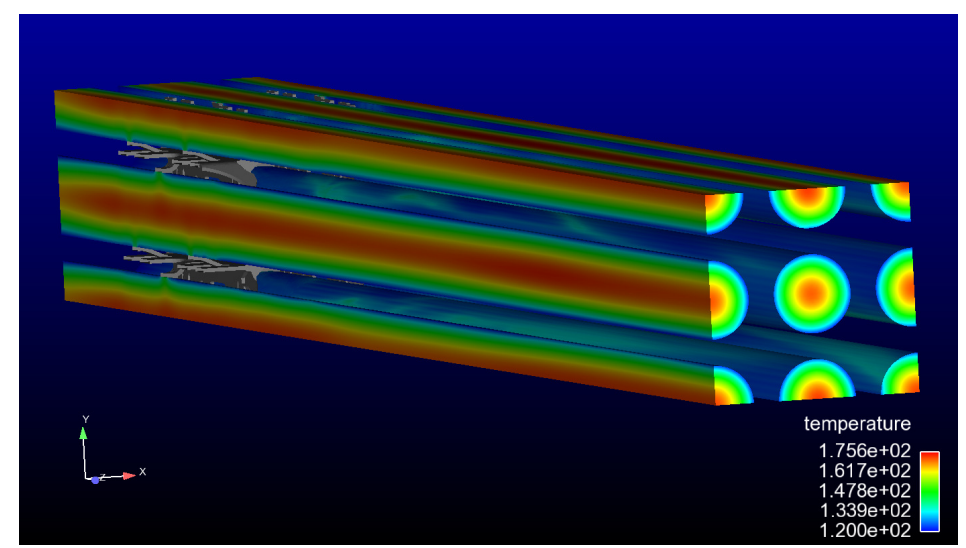

(b) Conjugate heat with constant volumetric heat source, $\operatorname{Pr}=1.42,2.6 \mathrm{M}$ element mesh.

Figure 1: Temperature contours single span 3x3 rod/spacer grid thermal fluid simulation, $R e=192,800$.

the stress tensor for a Newtonian fluid;

$$
\mathbf{T}=-\bar{p} \mathbf{I}+\tau+\tau_{t}=-\bar{p} \mathbf{I}+\mu_{\mathrm{eff}}\left[\nabla \overline{\mathbf{u}}+\nabla \overline{\mathbf{u}}^{T}\right]-\frac{2}{3} \mu_{\mathrm{eff}}(\nabla \bullet \overline{\mathbf{u}}) \mathbf{I}
$$

$\bar{p}$ is the isotropic hydrodynamic pressure, $\mu_{\mathrm{eff}}$ is the effective dynamic viscosity that can have contributions from molecular viscosity and a turbulent viscosity (e.g., $\mu_{\text {eff }}=\mu+\mu_{t}$ ), and $\mathbf{I}$ is the unity tensor. For the RANS model, the equations are closed by providing an estimate of the effective viscosity.

The heat flux vector is given by the Fourier model;

$$
\mathbf{q}=-\kappa_{\text {eff }} \nabla \bar{T}
$$

where $\kappa_{\text {eff }}=\kappa+\kappa_{t}$ is the effective thermal conductivity, $\kappa$ is the molecular coefficient of thermal conductivity $\kappa=\mu C_{p} / P r$, which can be specified by the specific heat capacity, 


\begin{tabular}{|c|c|}
\hline Governing Equations & $R_{P}=\nabla \cdot \overline{\mathbf{u}}=0$ \\
\hline Continuity & $R_{m}=\rho \frac{\partial \mathbf{u}}{\partial t}+\rho \overline{\mathbf{u}} \cdot \nabla \overline{\mathbf{u}}-\nabla \cdot \mathbf{T}=\mathbf{0}$ \\
\hline Momentum & $R_{T}=\rho C_{p} \frac{\partial \bar{T}}{\partial t}+\rho C_{p} \overline{\mathbf{u}} \cdot \nabla \bar{T}+\nabla \cdot \mathbf{q}=\mathbf{0}$ \\
\hline Energy
\end{tabular}

Table 1: Navier Stokes Equations.

dynamic viscosity and Prandtl number $\operatorname{Pr}$ and $\kappa_{t}$ is a turbulent conductivity. Similar to the momentum equation, the temperature equation is closed by providing a turbulent conductivity.

\subsection{Reynolds Average Navier-Stokes Turbulence Models}

In the Reynolds Averaged Navier-Stokes turbulence model methodology, a time filter is applied to the dependent flow variables resulting in the decomposition of the instantaneous velocity field into mean and fluctuating components,

$$
\mathbf{u}=\overline{\mathbf{u}}+\mathbf{u}^{\prime}
$$

and produces new equations that are very similar to the unfiltered equations with the addition of the Reynolds stress $\tau_{t}$,

$$
\tau_{t}=-\rho \overline{\mathbf{u}^{\prime} \otimes \mathbf{u}^{\prime}} .
$$

This new apparent stress arises mathematically through the filter operation applied to the nonlinear advection term. It is responsible for the modification of the flow field through turbulent fluctuations. In terms of numerical simulation, it represents the "closure problem" in that additional information or equations are now required that describe $\tau_{t}$ in order to close the filtered system of equations making solutions possible. Given a description of $\tau_{t}$, it is added to the "real" stresses in the original momentum equation, providing the necessary coupling. A common approach is to use the Boussinesq approximation where the Reynolds stresses are related to the filtered stresses through the following;

$$
\tau_{t}=-\rho \overline{\mathbf{u}^{\prime} \otimes \mathbf{u}^{\prime}} \approx \mu_{t}\left[\nabla \overline{\mathbf{u}}+\nabla \overline{\mathbf{u}}^{T}\right]-\frac{2}{3}\left[\mu_{t} \nabla \cdot \overline{\mathbf{u}}\right] \mathbf{I}
$$

where $\mu_{t}$ is the turbulent or eddy viscosity. Now the closure problem reduces to the task of calculating the eddy viscosity.

In a similar way, the averaged temperature equation contains the turbulent heat closure term, $\rho \overline{\mathbf{u}^{\prime} h^{\prime}}$, which includes the fluctuating enthalpy $h^{\prime}=C_{p} T^{\prime}$ and is modeled using the Boussinesq analogy;

$$
\mathbf{q}_{t}=-\rho \overline{\mathbf{u}^{\prime} h^{\prime}} \approx-\kappa_{t} \nabla \bar{T}
$$

where a turbulent or eddy conductivity is defined as;

$$
\kappa_{t}=\frac{\mu_{t} C_{p}}{P r_{t}}
$$


and $\mathrm{Pr}_{t}$ is the turbulent Prandtl number that is an unknown parameter in this problem. Commonly this term is estimated by the Reynolds analogy to be unity [22]. In section 5.2.2 we explore the sensitivity of the SARANS solution to this unknown parameter.

The equations listed in Table 1 represent both laminar and turbulent flows. For laminar flows, the effective viscosity and conductivity are given by the molecular values, and the turbulent viscosity and conductivity are zero. Thus the bar variables reduce to the exact laminar unknowns. In the simulation of turbulent flows, the viscosity coefficient in the viscous fluxes is replaced with the sum of the natural and eddy viscosity $\left(\mu_{t}+\mu\right)$, heat conductivity is replaced by the sum of natural and turbulent conductivity $\left(\kappa_{t}+\kappa\right)$ in equations $(1,2)$ and the turbulent Prandtl number $P r_{t}$ must be specified. To complete the RANS model equations, the task of specifying the eddy viscosity remains.

\subsection{Spalart-Allmaras Eddy Viscosity Model}

The Spalart-Allmaras turbulence model equation [40] for an incompressible fluid is given in Table 2. This equation is a convection-diffusion-reaction equation with a highly nonlinear stiff source term. The eddy viscosity is given by,

$$
R_{\hat{\nu}}=\rho \frac{\partial \hat{\nu}}{\partial t}+\rho \overline{\mathbf{u}} \cdot \nabla \hat{\nu}-\nabla \cdot\left(\rho\left(\frac{\nu+\hat{\nu}}{\sigma}\right) \nabla \hat{\nu}\right)-C_{b 1} \rho \hat{\nu} \hat{S}+C_{w 1} f_{w} \rho\left(\frac{\hat{\nu}}{d}\right)^{2}-\frac{C_{b 2} \rho}{\sigma}(\nabla \hat{\nu} \cdot \nabla \hat{\nu})=0
$$

Table 2: Spalart-Allmaras RANS Turbulence Model Equation.

$$
\mu_{t}=\rho \hat{\nu} f_{v 1}
$$

Functions defining the production and dissipation source terms in the model are listed below;

$$
\begin{array}{r}
\chi=\frac{\hat{\nu}}{\nu} \quad f_{v 1}=\frac{\chi^{3}}{\chi^{3}+C_{v 1}^{3}} \quad f_{v 2}=1-\frac{\chi}{1+\chi f_{v 1}} \\
f_{w}=g\left(\frac{1+C_{w 3}^{6}}{g^{6}+C_{w 3}^{6}}\right)^{1 / 6} \quad r=r+C_{w 2}\left(r^{6}-r\right) \quad r=\frac{\hat{\nu}}{\hat{S} k^{2} d^{2}} \\
\hat{S}=(2 \Omega \Omega)^{1 / 2}+\frac{\hat{\nu} f_{v 2}}{k^{2} d^{2}} \quad \Omega=\frac{1}{2}\left(\nabla \overline{\mathbf{u}}-\nabla \overline{\mathbf{u}}^{T}\right)
\end{array}
$$

where $\Omega$ is the rotation tensor. Model parameters are listed in Table 3. In the model,

\begin{tabular}{|c|c|c|c|c|c|c|c|c|}
\hline$\kappa$ & $C_{b 1}$ & $C_{b 2}$ & $\sigma$ & $C_{w 1}$ & $C_{w 2}$ & $C_{w 3}$ & $C_{v 1}$ & $C_{v 2}$ \\
\hline 0.41 & 0.1355 & 0.622 & $2 / 3$ & $\frac{C_{b 1}}{k^{2}}+\frac{1+C_{b 2}}{\sigma}$ & 0.3 & 2.0 & 7.1 & 5.0 \\
\hline
\end{tabular}

Table 3: Model parameters for Spalart-Allmaras turbulence model.

$\kappa$ is von Karman's constant, and $d$ appearing in the source terms represent the normal distance to the wall. At a solid wall, $\mu_{t}=0$, and therefore the boundary condition is, $\hat{\nu}_{w}=0$. A different formula for $\hat{S}$ has been proposed in Blazek [6] that prevents it from taking a value of zero. The modified term, $\hat{S}_{\text {mod }}$, is;

$$
\hat{S}_{\text {mod }}=f_{v 3}(2 \Omega \Omega)^{1 / 2}+\frac{\hat{\nu} f_{v 2}}{k^{2} d^{2}} \quad f_{v 2}=\left(1+\frac{\chi}{C_{v 2}}\right)^{-3} \quad f_{v 3}=\frac{\left(1+\chi f_{v} v 1\right)\left(1-f_{v 2}\right)}{\max (\chi, 0.001)}
$$


These modified terms are used in this paper.

\subsection{Normal Distance to the Wall}

The Spalart-Allmaras turbulence model described above requires the normal-distanceto-wall to evaluate the wall damping functions. The technique used in Drekar::CFD is presented by Tucker [41]. A distance scalar variable is solved from the equation listed in Table 4 . It has the boundary condition, $\phi_{w}=0$ at walls. The normal distance is then

$$
R_{\phi}=-\nabla^{2} \phi-1
$$

Table 4: Equation for Determining Normal-Distance-to-Wall.

determined from

$$
d=-\sqrt{\sum_{j=1,3}\left(\frac{\partial \phi}{\partial x_{j}}\right)^{2}} \pm \sqrt{\sum_{j=1,3}\left(\frac{\partial \phi}{\partial x_{j}}\right)^{2}+2 \phi}
$$

and equals the minimum absolute value of the two choices. This equation is solved once as a pre-processing step.

\section{Numerical Methods}

\subsection{Finite Element Discretization}

In this section we very briefly describe the stabilized finite element (FE) methods used for discretization of the Navier-Stokes equations with coupled transport. The governing PDEs for the flow and transport system are presented in Tables 1 and 2 along with the appropriate closure models that are required to compute turbulent transport effects. For this incompressible form of the Navier-Stokes system the stabilized FE method allows equal order interpolation of velocity and pressure and also provides stabilization of the convection operators to limit oscillations due to high grid Reynolds and Peclet number effects $[7,21,12]$. In the present application piecewise linear Lagrange basis and Gaussian quadrature are used to construct the discretized residual equations.

Our formulation is capable of handling incompressible, low Mach number, variable density (temperature and chemical species dependent), and low speed compressible flows. This flexibility is enabled by the fully-implicit time integration and fully-coupled nonlinear solver technology that is described later in Section 3.2. In terms of spatial discretization the most challenging aspects are associated with the incompressible limit. In this case a mixed Galerkin FE formulation requires discrete spaces that satisfy the Ladyzhenskaya-Babuska-Brezzi (LBB) condition; (see e.g., [20]). Among other things, this condition prevents the use of equal order finite element spaces, defined with respect to the same partition of the computational domain into finite elements. Linearization of the mixed nonlinear equations also leads to indefinite linear systems that are more difficult to solve by iterative methods. An additional difficulty is that the mixed Galerkin formulation is prone to instabilities for highly convected flows, even if the LBB condition is satisfied by the finite element spaces. 
Stabilized finite element methods ([7, 21, 12]) address these issues by using properly weighted consistent residuals of the momentum equation to simultaneously relax the incompressibility constraint and add streamline-diffusion and nonlinear-discontinuitycapturing operators (DCO) to the weak equations. A significant added advantage of stabilization is that the linearized problems are real positive definite and therefore performance of iterative solvers can be improved [4]. Finally this method allows equal-order interpolation, and thus enables the simplification of data structures for a general parallel unstructured FE code and the linear algebra interface for iterative solution methods $[34,32,23]$. The FE weak form for the governing PDEs (Tables 1 and 2) are presented in Equations 10 - 13.

To improve stability for highly convected flows we can also employ discontinuitycapturing operators that provide additional isotropic, or anisotropic crosswind, diffusion to supplement the natural streamline upwind Petrov-Galerkin (SUPG) and pressure stabilized Petrov-Galerkin (PSPG) contributions of the stabilized formulation. The resulting finite element formulation decreases numerical oscillations and allows for stable and accurate finite element solutions when the cell Reynolds, $R e_{c}$, and thermal energy and mass transport Peclet numbers, $P e_{c}$, are greater than one.

- Momentum:

$$
\begin{gathered}
F_{\mathbf{u}_{\mathbf{i}}}=\int_{\Omega} R_{m_{i}} \Phi d \Omega+\sum_{e} \int_{\Omega^{e}} \rho \tau_{m}(\mathbf{u} \cdot \nabla \Phi) R_{m_{i}} d \Omega+\sum_{e} \int_{\Omega^{e}} \rho \tau_{c}[\nabla \Phi]_{i} R_{P} d \Omega \\
+\int_{\Omega^{e}} \nu_{m, i} \nabla \Phi \cdot \mathbf{C} \nabla \mathbf{u}_{i} d \Omega
\end{gathered}
$$

- Continuity:

$$
F_{P}=\int_{\Omega} R_{P} \Phi d \Omega+\sum_{e} \int_{\Omega^{e}} \rho \tau_{m} \nabla \Phi \cdot \mathbf{R}_{\mathbf{m}} d \Omega
$$

- Temperature:

$$
F_{T}=\int_{\Omega} R_{T} \Phi d \Omega+\sum_{e} \int_{\Omega^{e}} \rho C_{p} \tau_{T}(\mathbf{u} \cdot \nabla \Phi) R_{T} d \Omega+\int_{\Omega^{e}} \nu_{T} \nabla \Phi \cdot \mathbf{C} \nabla T d \Omega
$$

- Spalart-Allmaras RANS Turbulence Model:

$$
F_{\hat{\nu}}=\int_{\Omega} R_{\hat{\nu}} \Phi d \Omega+\sum_{e} \int_{\Omega^{e}} \rho \tau_{\hat{\nu}}(\mathbf{u} \cdot \nabla \Phi) R_{\hat{\nu}} d \Omega+\int_{\Omega^{e}} \nu_{\hat{\nu}} \nabla \Phi \cdot \mathbf{C} \nabla \hat{\nu} d \Omega
$$

In these equations the stability parameters (the $\tau$ 's) are functions of the fluid velocity, $\mathbf{u}$ and the transport coefficients of the PDEs and are given in Table 5. Here, the last integral operator on the R.H.S. of the Momentum, Temperature and Spalart-Allmaras weak equations is a cross-stream oriented tensor that adds diffusion in the cross-stream 
direction, orthogonal to the SUPG contribution. It should be pointed out that the DCO coefficients (e.g., $\nu_{T}$ ) are proportional to the PDE residual and therefore represent a consistent modification of the equations that the exact solution satisfies.

The specific definition of the intrinsic-time-scale stability parameters are provided in Table 5 for momentum, continuity, temperature and SARANS equations. The specific form of the intrinsic time scales $\tau$ 's are an adaptation of the quadratic form suggested by Shakib [38] for the Navier-Stokes equations with coupled scalar transport. It should

\begin{tabular}{|c|c|}
\hline Momentum & $\tau_{m}=\left(\left(\frac{\rho}{\Delta t}\right)^{2}+\rho^{2} \mathbf{u} \mathbf{G}_{c} \mathbf{u}+12 \mu^{2}\left\|\mathbf{G}_{c}\right\|\right)^{-1 / 2}$ \\
\hline Continuity & $\tau_{c}=\left(8\left\|\mathbf{G}_{c}\right\| \tau_{m}\right)^{-1}$ \\
\hline Temperature & $\tau_{T}=\left(\left(\frac{\rho C_{p}}{\Delta t}\right)^{2}+\left(\rho C_{p}\right)^{2} \mathbf{u} \mathbf{G}_{c} \mathbf{u}+12 \lambda^{2}\left\|\mathbf{G}_{c}\right\|\right)^{-1 / 2}$ \\
\hline SARANS & $\tau_{\hat{\nu}}=\left(\left(\frac{\rho}{\Delta t}\right)^{2}+\rho^{2} \mathbf{u} \mathbf{G}_{c} \mathbf{u}+12\left(\mu_{\mathrm{eff}}\right)^{2}\left\|\mathbf{G}_{c}\right\|\right)^{-1 / 2}$ \\
\hline
\end{tabular}

Table 5: Intrinsic-time-scale stability parameters for transport equations.

be pointed out that the multidimensional effect of convection is incorporated into the stability parameters by the use of the contravariant metric tensor, $\mathbf{G}_{c}$ (Equation (14)), of the transformation from local element coordinates $\left\{\zeta_{\alpha}\right\}$ to physical coordinates $\left\{x_{i}\right\}$,

$$
\left[\mathbf{G}_{c}\right]_{i j}=\frac{\partial \zeta_{\alpha}}{\partial x_{i}} \frac{\partial \zeta_{\alpha}}{\partial x_{j}} .
$$

Shakib [38] considers the one dimensional limiting case of this multidimensional definition for the advection-diffusion equation and presents a comparison with the original SUPG technique. The anisotropic discontinuity capturing operator defined for the scalar component of velocity, $u_{i}$, is;

$$
\nu_{m, i}=\max \left\{0, \chi \frac{\operatorname{diam}\left(\Omega^{e}\right)\left|\mathbf{R}_{m, i}\right|}{2\left\|\nabla \mathbf{u}_{i}\right\|_{2}}-\mu\right\},
$$

and the cross-wind orthogonal projection, $\mathbf{C}$, to the velocity vector, $\mathbf{u}$, is given by,

$$
\mathbf{C}=\mathbf{I}-\frac{\mathbf{u} \otimes \mathbf{u}}{\|\mathbf{u}\|_{2}^{2}} .
$$

Similar definitions are used for each of the scalar components for which the discontinuity operators are defined. The constant, $\chi$, is a user defined value that we commonly take as $\chi=1 / 2$ and currently we approximate the $\operatorname{diam}\left(\Omega^{e}\right)$ by the norm of the metric tensor, (i.e., $\left.\operatorname{diam}\left(\Omega^{e}\right) \approx 1 / \sqrt{\left\|\mathbf{G}_{c}\right\|}\right)$. In the computations for this study we do not require the inclusion of the DC operators.

\subsection{Overview of Computational Solution Methods}

\subsubsection{Fully-implicit Time Integration and Strongly-coupled Newton-Krylov Solver}

The incompressible flow equations in Tables 1 and 2 can be time integrated with fully-implicit methods (e.g., BDFn and SDIRK type methods [36, 37]), or a pseudotransient strategy (using BDF1) when the goal is to reach a steady-state for highly nonlinear solutions. Additionally, direct-to-steady-state solutions are possible when the 
nonlinearity is less-strong or a very good initial guess is available from a previous nearby solution or continuation methods are employed [32].

The governing equations discretized by the finite element method result in a very large-scale, coupled highly nonlinear algebraic system(s) that must be solved. Solution of these systems places a heavy burden on both the nonlinear and linear solvers and require robust, scalable, and efficient methods. In this study Newton-based iterative nonlinear solvers ([11]) are employed. These solvers can exhibit quadratic convergence rates independent of problem size when sufficiently robust linear solvers are available. For the latter, we employ Krylov iterative techniques. A Newton-Krylov (NK) method is an implementation of Newton's method in which a Krylov iterative solution technique is used to approximately solve the linear systems, $\mathbf{J}_{\mathbf{k}} \mathbf{S}_{\mathbf{k}+\mathbf{1}}=-\mathbf{F}_{\mathbf{k}}$, that are generated at each step of Newton's method. For efficiency, an inexact Newton method ([11]; [13]) is usually employed, whereby one approximately solves the linear systems generated in the Newton method by choosing a forcing term $\eta_{k}$ and stopping the Krylov iteration when the inexact Newton condition, $\left\|\mathbf{F}_{\mathbf{k}}+\mathbf{J}_{\mathbf{k}} \mathbf{S}_{\mathbf{k}+\mathbf{1}}\right\| \leq \eta_{\mathbf{k}+\mathbf{1}}\left\|\mathbf{F}_{\mathbf{k}}\right\|$ is satisfied. The particular Krylov method that is used in this study is a robust non-restarted GMRES method that is capable of iteratively converging to the solution of very large non-symmetric linear systems such as developed from the discretized equations, provided a sufficiently robust and scalable preconditioning method is available [36, 37].

\subsubsection{Fully-coupled Algebraic Multilevel Preconditioner}

A scalable preconditioner for the iterative linear solver is necessary to achieve solutions efficiently. For this reason, a fully-coupled algebraic multigrid method (AMG) is employed [18]. In general AMG methods are significantly easier to implement and integrate with complex unstructured mesh discretizations than geometric multigrid methods [42, 35, 32]. The fully-coupled AMG preconditioner and the Newton-GMRES method described above have been demonstrated to provide a robust and scalable nonlinear/linear iterative solution strategy for a number of applications that include CFD, transportreaction systems and MHD (see e.g., [24,37] and the references contained there in). While this current study is not focused on parallel performance of the strongly coupled solution methods described above, each of the computations used parallel execution to provide fast execution times for the multiple forward and adjoint solutions. In general the runs in this study used between 64 to 1024 cores for execution of the smaller forward problems and/or larger higher-order adjoint solves. The ability to carry out efficient parallel solutions is important. For example in the case were $140+$ solutions of a moderate sized 3D RANS turbulence computation is carried out for construction of surrogate models for development of a global sensitivity analysis in Section 5.2.3. The detailed reporting required to document the performance and scalability of the methods for both the forward and adjoint solves is beyond the scope of this current study and will be considered for an upcoming study of these methods applied to resistive magnetohydrodynamic simulations. Finally we note that the forward solvers have been recently demonstrated to scale to over $10^{5}$ cores and solve problems with over $10^{9}$ unknowns [37].

\subsubsection{Nonlinear and Linear Convergence Criteria}

To complete the description of the numerical method that is employed in this study the convergence criteria that are used to determine convergence of the nonlinear/linear 
iterative methods must be stated. Two nonlinear solver convergence criteria are employed. The first is a requirement of sufficient reduction in the relative nonlinear residual norm, e.g., $\left\|F_{k}\right\| /\left\|F_{o}\right\|<10^{-2}$. In general, this requirement is set to be easily satisfied. The second convergence criterion is based on a sufficient decrease of a weighted norm of the relative size of the Newton update vector. The latter criterion requires that the correction, $\Delta \chi_{i}^{k}$, for any grid point unknown, $\chi_{i}$, is small compared to its magnitude, $\left|\chi_{i}^{k}\right|$, and is given by

$$
\sqrt{\frac{1}{N_{u}} \sum_{i=1}^{N_{u}}\left[\frac{\left|\Delta \chi_{i}\right|}{\varepsilon_{r}\left|\chi_{i}\right|+\varepsilon_{a}}\right]^{2}}<1 .
$$

Here $N_{u}$ is the total number of unknowns, $\varepsilon_{r}$ is the relative error tolerance between the variable correction and its magnitude, and $\varepsilon_{a}$ is the absolute error tolerance of the variable correction, which sets the magnitude of components that are to be considered to be numerically zero. In this study, the relative-error and absolute-error tolerance are $10^{-5}$ and $10^{-8}$ respectively for our verification studies of adjoint-based methods. In practice this criteria controls convergence of the nonlinear solver to sufficient accuracy. Finally in each Newton sub-step the linear system is solved to a relative tolerance of $\eta=10^{-6}$ with the knowledge that the overall accuracy of the solution for each time step is controlled as described above for convergence of the Newton step.

\subsection{Embedded Uncertainty Quantification for Thermal Hydraulics}

In this section we describe the use of embedded algorithms to estimate errors in a given Quantity of Interest (QoI), compute parameter sensitivities and construct surrogate models for UQ statistics using computed sensitivities. The embedded algorithms make use of the same operators, tools and data structures that are used to solve the forward problem, namely, Jacobian matrix operator, weak residual operator, and automatic differentiation. Several CASL Verification \& Uncertainty Quantification (VUQ) milestone reports related to this work have been written (see Wildey et al. [43], [44]), and a detailed description of this approach can be found in $[3,5,10]$. In these milestone reports adjoint based error estimators were derived and several examples including steady-state turbulent flow simulations using the Spalart-Allmaras model were presented. In this paper, parameter sensitivities and their application to UQ are also included.

As an example, a QoI $g(u)$ may be defined as;

$$
g(u)=\frac{1}{\operatorname{Vol}(\omega)} \int_{\Omega} u \delta d \Omega,
$$

which represents the average value within a subdomain $\omega \subset \Omega$. In this functional, $u$ is the solution vector. The function $\delta$ is the characteristic function that is one for $x \in \omega$ and zero otherwise.

Abstractly the solution to the forward problem, $u$ satisfies

$$
\mathcal{L}(u ; \mathbf{p})=\mathbf{f}(\mathbf{p})
$$

where $\mathcal{L}$ is the nonlinear PDE operator, $u$ is the solution, $\mathbf{f}$ is the source function, and $\mathbf{p}$ is a vector of parameter values. The residual is defined as

$$
\mathcal{R}(u ; \mathbf{p}):=\mathcal{L}(u ; \mathbf{p})-\mathbf{f}(\mathbf{p}) .
$$


The dual problem defines the adjoint function $\psi$

$$
\left(\mathcal{L}^{\prime}(u ; \mathbf{p})\right)^{*} \psi=\partial_{u} g(u, \mathbf{p}) .
$$

Using the definition of the adjoint and the residual, computable expressions for the error and the parameter sensitivities in the QoI can be derived:

$$
\begin{aligned}
g(u ; p)-g(U ; \mathbf{p}) \approx(\psi, \mathcal{R}(u ; \mathbf{p})), & (\text { Error }) \\
\partial_{p} g(U ; \mathbf{p})=\left(\psi, \partial_{p} \mathcal{R}(u ; \mathbf{p})\right), & (\text { Sensitivity })
\end{aligned}
$$

where $U \approx u$.

An algorithm for computing error estimates for a QoI is presented next (taken from [43]). Define low and high order approximation spaces $V_{L}$ and $V_{H}$ respectively. We will refer to low- and high-order approximations. In this paper $V_{L}$ is defined by the space of piecewise linear polynomials on the mesh, $V_{H}$ is the space of piecewise quadratic polynomials on the same mesh. Given an approximate solution $u_{L} \in V_{L}$ satisfying Equation 18 the algorithm to compute error estimates and sensitivities proceeds in the following steps:

1. Extend the low-order approximation into the higher order space:

$$
\mathcal{P}\left(u_{L}\right)=u_{H} .
$$

2. Compute a high-order residual:

$$
\mathbf{r}_{H}=\mathcal{R}\left(u_{H}, \mathbf{p}\right) .
$$

Because this is a high order residual, Galerkin orthogonality is not a problem and the vector $\mathbf{r}_{H}$ may not be zero.

3. Build a high-order Jacobian operator $\mathbf{J}_{H}$ of the $\mathcal{R}$ operator linearized around $u_{H}$.

4. Solve the adjoint problem in the high order space:

$$
\mathbf{J}_{H}^{T} \psi_{H}=\frac{\partial g}{\partial u} .
$$

The transpose may represent taking the strong-form adjoint (see [10] for more discussion).

5. Finally, estimate the error in the QoI using (see Eq. 21):

$$
\left(\psi_{H}, \mathcal{R}\left(u_{H} ; \mathbf{p}\right)\right) .
$$

6. Optionally compute the sensitivity in the QoI using (see Eq. 22):

$$
\left(\psi_{H}, \partial_{p} \mathcal{R}\left(u_{H} ; \mathbf{p}\right)\right) .
$$

Alternatively, the QoI sensitivity can be computed using the low-order solution $u_{L}$. In this case solving the adjoint problem in a high-order space is not necessary. The adjoint problem could instead be solved in the low-order space $V_{L}$. In this case the extension of $u_{L}$ to high order is unnecessary. In the computations presented in this paper only low-order sensitivities are computed. 
We note that the procedure described above to compute an adjoint solution is a discreteadjoint approach where the discrete adjoint operator is applied to the forward Jacobian. We have previously studied the effects of the discrete adjoint approach on the accuracy of error estimates for stabilized finite element formulations [10], but we did not observe any issues with the examples considered in this paper. Moreover, we remark that while this is a discrete adjoint approach, we do not utilize a formal Algorithmic Differentiation, or reverse-mode $\mathrm{AD}$, to compute the adjoint solution. While such an approach has certain advantages in the calculation of sensitivities, it is quite complicated to implement for fully-implicit simulations using Newton-Krylov solvers and is not particularly ameneable towards error estimation which typically requires approximation of the adjoint solution in a higher-order space.

\subsection{Brief Overview of the Use of Automatic Differentiation}

An important algorithmic design aspect of the Drekar::CFD code is the use of templatebased embedded automatic differentiation technology to enable the implementation of Newton based nonlinear solvers and adjoint-based error-estimation and sensitivity techniques (see $[28,29]$ for a detailed description of $\mathrm{AD}$ and the breadth of solution and analysis algorithms that it can enable). In our implementation template-based embedded AD enabled by the Trilinos/SACADO package is used for automated construction of the Jacobian operator required for the fully-coupled implicit forward solution (Section 3.2.1), and the transposed Jacobian operator for the adjoint equations (Section 3.4). Additionally, AD is used to produce the derivatives of QoI (responses) with respect to parameters that are required for the right hand side in the adjoint equations (Eq. 20) and the derivatives of the residual equations required in the computation of the sensitivities (Eq. 22). This AD technology gives developers the capability to rapidly implement new models, while easily maintaining the implicit solution approach with mathematically accurate Jacobians. In order to fascilitate this AD technology, we have diligently developed models that are ameneable to $\mathrm{AD}$, i.e., models that are differentiable with respect to the forward solution and parameters. As stated above we believe the AD technology is also critical in the context of sensitivity analysis. The set of possible scientific QoI and uncertain parameters required by an analyst may represent a fairly large space of possible use cases. AD technology combined with the directed acyclic graph employed in Drekar::CFD (see [26] for description), allows for QoI and parameters to be specified at runtime while maintaining the ability to compute exact sensitivities (up to machine precision). In the absence of such a tool an analyst would be subject to hand coding sensitivities for every QoI/parameter pair, resulting in a substantial increase in development time and difficulty.

Finally we briefly comment on the memory usage for the AD implementation. In our implementation of using $\mathrm{AD}$ we have embedded the computation of the derivatives at the element level where the structure of the FE unknown connectivity is known and available and is most efficiently processed in terms of computation time and memory usage. At this level a set of small dense element matrices contain the entries for the Jacobian and after the element integration loop it is then scattered to a global distributed sparse matrix data structure. This procedure does not incur substantially more memory usage since we do not use $\mathrm{AD}$ to differentiate the entire residual vector with respect to the FE nodal unknowns. Instead AD is only applied on the element level integration which efficiently 
limits the computations to the unknowns that interact to influence the residual at a particular FE node.

\subsection{Drekar::CFD}

An implementation of the governing equations discretized by the numerical methods discussed above and including an embedded adjoint solver is contained in the code called Drekar::CFD [30]. It was developed at Sandia National Laboratories with partial funding from the CASL program and relies heavily on the Sandia software called Trilinos[17] to which a number of the authors of this paper have contributed for many years.

\section{Verification of Methods}

\subsection{Nusselt Number in Fully Developed Pipe Flow}

In heat transfer problems where heat is transfered from a boundary into a fluid or vice versa, the Nusselt number $(N u)$ represents the ratio of convective to conductive heat transfer. Kays\&Crawford [22] have derived expressions for the $N u$ for fully developed pipe flow, both laminar and turbulent. For laminar flow theoretical values for constant wall temperature and constant heat flux wall boundary conditions have been derived. With these analytic expressions available, this flow problem is used to verify the computational thermal hydraulic models. In this section, we review the procedure for computing $N u$ for fully developed pipe flow and compare the semi-analytic values from Kays\&Crawford [22] with computed values from Drekar::CFD solutions. In this context fully developed means the velocity field is stationary and independent of axial location and the temperature field has evolved in the axial direction to a scale similar profile independent of axial location. The distance required to reach that location is a function of Prandtl and Reynolds number and is referred to as the thermal entry length.

\subsubsection{Laminar Flow}

Consider fully developed flow in a three-dimensional circular pipe. The flow is considered fully developed when the radial velocity profile does not change with axial location in the pipe. This condition is achieved through periodic inflow-outflow boundary conditions and applying a momentum source term to force the flow in the axial direction.

The Nusselt number is defined as;

$$
N u=\frac{h D}{\kappa}
$$

where $h$ is the local convection heat transfer coefficient, $D$ is the pipe diameter and $\kappa$ is the molecular thermal conductivity coefficient. The mass averaged velocity $V$ and mixing temperature $T_{m}$ are the average velocity and temperature for a cross-section of the pipe defined as;

$$
T_{m}=\frac{1}{\rho V A} \int_{A} \rho \mathbf{u} \cdot \mathbf{n} T d A \quad V=\frac{1}{\rho A} \int_{A} \rho \mathbf{u} \cdot \mathbf{n} d A,
$$

where $A$ is the cross-sectional area, and $\mathbf{u}$ is the velocity vector at a point on the crosssection surface and $\mathbf{n}$ is a unit vector normal to the surface. Newton's law of cooling can be stated as;

$$
\dot{q}_{w}^{\prime \prime}=h\left(T_{w}-T_{m}\right)
$$


where $\dot{q}_{w}^{\prime \prime}$ is the average pipe wall heat flux for a single axial location. The wall heat flux and temperature $T_{w}$ are defined as;

$$
\dot{q}_{w}^{\prime \prime}=\frac{1}{L} \oint_{L} \kappa \frac{\partial T}{\partial \mathbf{n}} d l \quad T_{w}=\frac{1}{L} \oint_{L} T d l
$$

In this case $\frac{\partial T}{\partial \mathbf{n}}$ is the temperature gradient normal to the wall and tangent to the surface for which the flux is computed and the integration is a line traversing the circumference of the pipe $L$.

The final definition of $N u$ is;

$$
N u=\frac{h D}{\kappa}=\frac{\dot{q}_{w}^{\prime \prime} D}{\kappa\left(T_{w}-T_{m}\right)} .
$$

Drekar::CFD solutions with $\operatorname{Pr}=7$ and Reynolds number based on mean axial velocity and pipe diameter, $\operatorname{Re}_{D}=\frac{\rho V D}{\mu}=10$, were obtained by solving direct-tosteady-state on a mesh containing 344,064 elements. For this large Prandtl number, representative of water, the near wall resolution requirement is seven times greater for the thermal boundary layer than for the momentum boundary layer. Therefore, a very fine mesh was used to capture the thermal boundary layer. The tube diameter is $(D=$ $0.01 \mathrm{~m}$ ). Two views of a typical computational mesh are shown in Figure 2. The pipe axis is aligned with the $\mathrm{x}$-direction. Constant density, viscosity and conductivity
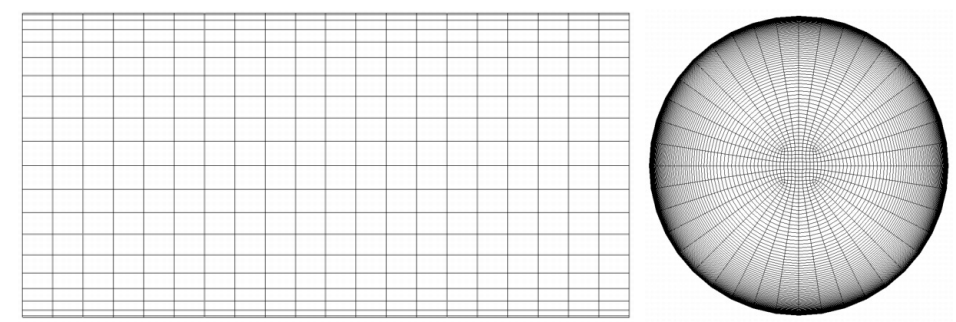

Figure 2: Two cut-away views of a 62,000 element pipe mesh [33].

are specified for these simulations, thus, the momentum and temperature equations are decoupled when the flow is laminar. This allows us to solve the momentum equations first using periodic inflow and outflow boundary conditions without solving for temperature. In these simulations, the initial velocity profile is parabolic with respect to the pipe centerline. These conditions correspond to the analytic solution for velocity. Next, the temperature equation is solved by holding the velocity field fixed. The inflow temperature is constant and so the thermal boundary layer grows in the downstream direction. The Nusselt number is derived assuming fully developed velocity and temperature fields. Therefore, the pipe must be longer than the thermal entry length which is defined as the distance required for the temperature boundary layer to reach a point where the cross-section profile no longer varies with stream-wise location. Kays\&Crawford derive the thermal entry length for laminar flow as;

$$
\frac{x}{D}=0.05 \operatorname{Pr} \operatorname{Re}_{D}
$$


for both constant temperature and constant heat flux wall boundary conditions. For the conditions presented here, $\frac{x}{D}=3.5$. The computational domain was $x=20 D$ and $\mathrm{Nu}$ was computed at $x=10 D$.

The analytic values of Nusselt number for fully developed laminar pipe flow for constant wall heat flux and constant temperature are presented in Table 6 along with predictions using Drekar::CFD. The analytic values are independent of $\operatorname{Pr}$ and $R e_{D}$. The

\begin{tabular}{|c|c|c|}
\hline & $\begin{array}{c}\mathrm{Nu} \\
\left(\text { const. } \dot{q}_{w}^{\prime \prime}\right)\end{array}$ & $\begin{array}{c}\mathrm{Nu} \\
\text { (const. } T_{w} \text { ) }\end{array}$ \\
\hline \hline K\&C & 4.364 & 3.658 \\
\hline Drekar::CFD & 4.430 & 3.682 \\
\hline$\% \Delta$ & $1.5 \%$ & $0.6 \%$ \\
\hline
\end{tabular}

Table 6: Fully developed laminar pipe flow, $\mathrm{Nu}$ (const. heat flux), $\mathrm{Nu}$ (const. temperature), $R e=10$, $\operatorname{Pr}=7.0$.

percent difference between analytic and numerical values are also shown.

\subsection{Error Estimation and Adjoint Sensitivity Analysis for CFD: Fully-Developed 3D Rectangular Laminar Duct Flow}

In this first example of adjoint based error estimation and sensitivity analysis the fully developed steady laminar flow in a rectangular cross-sectional duct is simulated. For this problem, an analytic solution exists;

$$
u(y, z)=\frac{16 a^{2}}{\mu \pi^{3}}\left(-\frac{\partial p}{\partial x}\right) \sum_{i=1,3,5, \ldots}^{\infty}(-1)^{(i-1) / 2}\left[1-\frac{\cosh (i \pi z / 2 a)}{\cosh (i \pi b / 2 a)}\right] \cdot \frac{\cos (i \pi y / 2 a)}{i^{3}} .
$$

For a QoI, the average $x$-component of velocity $(\mathrm{Vx})$ is chosen;

$$
\bar{V}=\int_{-a}^{a} \int_{-b}^{b} V_{x} d y d z
$$

An analytic solution for this QoI also exists;

$$
\bar{V}=\frac{a^{2}}{3 \mu}\left(-\frac{\partial p}{\partial x}\right)\left[1-\frac{192 a}{\pi^{5} b} \sum_{i=1,3,5, \ldots}^{\infty} \frac{\tanh (i \pi b / 2 a)}{i^{5}}\right] .
$$

In this verification problem the reference solution was computed using 15 terms in the series. The forward solution was approximated using SUPG with Q1 elements and the adjoint equation was solved with Q2 elements. The computational domain contained $N x \times N y \times N z=100 \times 40 \times 40$ elements in the mesh. Solutions were computed at four Reynolds numbers $R e=\frac{\rho V L}{\mu}$ and the solutions were obtained by starting from a trivial initial guess with a direct-to-steady-state solution procedure. Parameters in this simple numerical sensitivity study were dynamic viscosity and pressure gradient $\left(\mu, G_{0}\right)$ respectively. Figure 3 shows a surface colored by magnitude of $x$-component of velocity. Table 7 shows a comparison between the exact QoI and the Drekar::CFD predicted QoI 
and the adjoint based error estimate for four Reynolds number solutions. The fourth column is the effectivity which is the ratio of the adjoint based error estimate and the true error (difference between exact and Drekar::CFD predicted QoI). Table 8 compares the sensitivity of the QoI to the viscosity and pressure at the four Reynolds number solutions. The exact sensitivity is obtained by differentiating the analytical solution (Eq. 36) of the QoI with respect to either viscosity or pressure gradient. Figure 4 shows

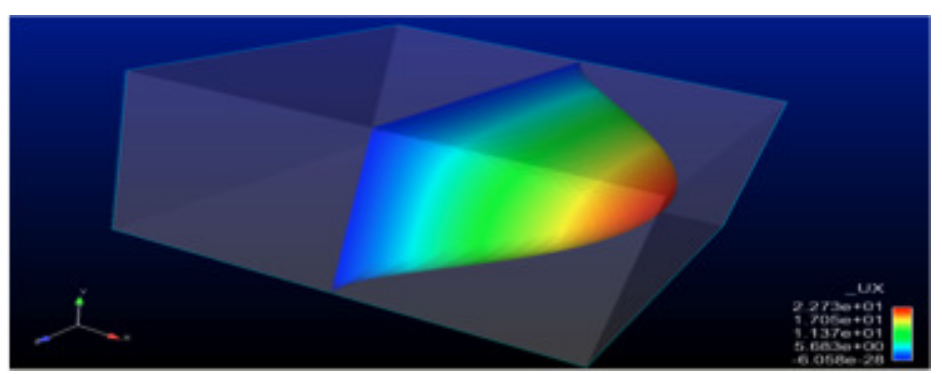

Figure 3: $x$-component of velocity colored by the magnitude in a rectangular duct.

\begin{tabular}{|c|c|c|c|c|}
\hline Re & $\begin{array}{c}\text { QoI } \\
\text { Exact) }\end{array}$ & $\begin{array}{c}\text { QoI } \\
\text { (Drekar::CFD) }\end{array}$ & $\begin{array}{c}\text { Adj. } \\
\text { Est. Err. }\end{array}$ & Eff \\
\hline \hline 50 & 23.4292 & 23.4077 & $2.15 \mathrm{e}-2$ & 1.01 \\
\hline 150 & 70.2875 & 70.2230 & $6.55 \mathrm{e}-2$ & 1.02 \\
\hline 500 & 234.292 & 234.077 & $2.18 \mathrm{e}-1$ & 1.01 \\
\hline 1000 & 468.583 & 468.154 & $4.36 \mathrm{e}-1$ & 1.02 \\
\hline
\end{tabular}

Table 7: Error Estimation for QoI at different Re for a rectangular duct.

\begin{tabular}{|c|c|c|c|c|c|c|}
\hline $\operatorname{Re}$ & $\begin{array}{c}d Q o I / d \mu \\
\text { Exact) }\end{array}$ & $\begin{array}{c}d Q o I / d \mu \\
\text { (Drekar::CFD) }\end{array}$ & $\begin{array}{c}\text { Error In } \\
\text { Sens. Est. }\end{array}$ & $\begin{array}{c}d Q o I / d G_{0} \\
\text { (Exact) }\end{array}$ & $\begin{array}{c}d Q o I / d G_{0} \\
\text { (Drekar::CFD) }\end{array}$ & $\begin{array}{c}\text { Error In } \\
\text { Sens. Est. }\end{array}$ \\
\hline \hline 50 & -7.8095 & -7.8026 & $0.09 \%$ & $-4.686 \mathrm{e}-2$ & $-4.686 \mathrm{e}-2$ & $0.0015 \%$ \\
\hline 150 & -70.285 & -70.223 & $0.09 \%$ & -0.14057 & -0.14058 & $0.0014 \%$ \\
\hline 500 & -780.97 & -780.26 & $0.09 \%$ & -0.46858 & -0.46859 & $0.0015 \%$ \\
\hline 1000 & -3121.0 & -3121.0 & $0.09 \%$ & -0.93717 & -0.93718 & $0.0014 \%$ \\
\hline
\end{tabular}

Table 8: Adjoint Sensitivity Analysis for two QoI at different Re for a rectangular duct.

convergence with mesh spacing for the exact error in the QoI, adjoint error estimate in the $\mathrm{QOI}(\mathrm{Vx})$, and the exact relative error in adjoint sensitivity to viscosity $(d Q o I / d \mu)$ for $R e=500$. The results show the error-estimate and the exact errors are converging at a rate very close to second-order. 


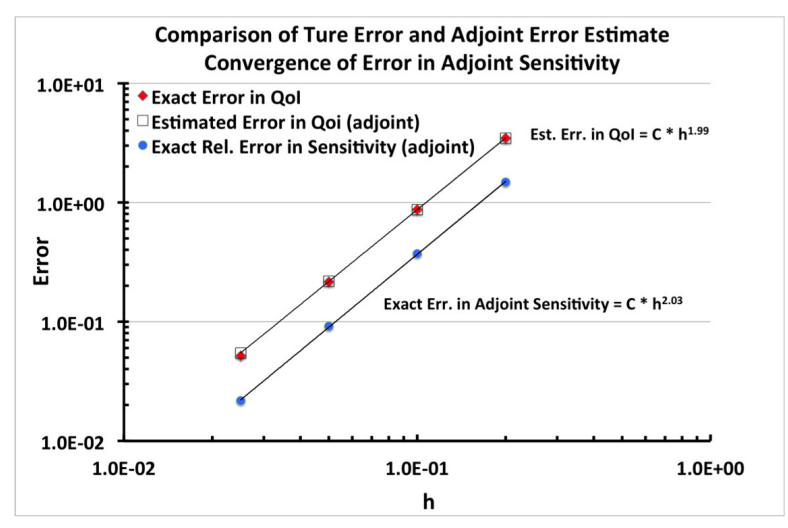

Figure 4: Convergence of exact error in QoI, adjoint based estimated error in QoI, and exact relative error in sensitivity.

\section{Results and Discussion}

\subsection{Swirling Flow Through a Cylindrical Pipe with Sudden Expansion}

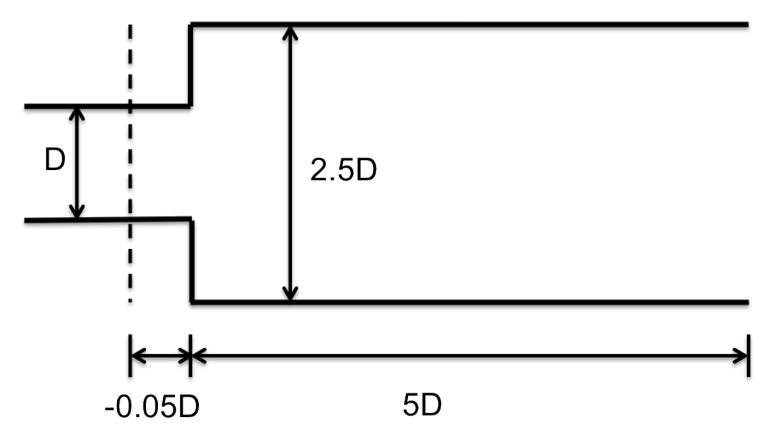

Figure 5: Benchmark test case \#2 expansion tube sketch. The dotted line represents the inlet plane fo the computational domain.

This challenging swirling turbulent flow problem is a standard CASL test case that is labeled as Test Case \#2 [27, 25]. It models a turbulent flow in a circular pipe through a sudden expansion with and without initial swirl induced at the pipe inlet. A sketch of the geometry is shown in Figure 5 . Here, the pipe surfaces are assumed to be smooth and the expansion is abrupt. A pipe with diameter $D$ and radius $R_{1}$ terminates into a pipe with diameter $2.5 D$ and radius $R_{2}$. Flow is in the positive $z$ direction. The inlet is placed at $z=-0.05 D$, the expansion plane at $z=0$ and the outlet is at $z=5 D$. The benchmark specification has the outlet at $z=1.825 \mathrm{D}$. However, this proved to be too short causing a significant modification to the flow-field due to the outflow boundary condition. A swirl number $S$ is defined as the ratio of angular momentum flux to linear momentum flux divided by a reference radius [25]. For this axisymmetric configuration the inflow 
velocity vector is described by an axial component $V_{z}$ and an asmuthal component $V_{\theta}$. $V_{\theta}=\omega r$ is specified as solid body rotation. Both $U_{z}$ and $\omega$ are constant. Given this definition for the inlet velocity, $S$ is defined as;

$$
S=\frac{\omega R_{1}}{2 U_{z}} .
$$

The flow is specified by $R e_{D}$ and $S$. The walls are assumed to be no-slip. At the outflow boundary, a zero stress condition is assumed that sets the normal component of the stress traction vector to zero at the surface. In the orthogonal directions the tangential components of velocity are set to zero. A standard outflow natural condition on the SARANS equations is also assumed. Two views of the three-dimensional 141,568
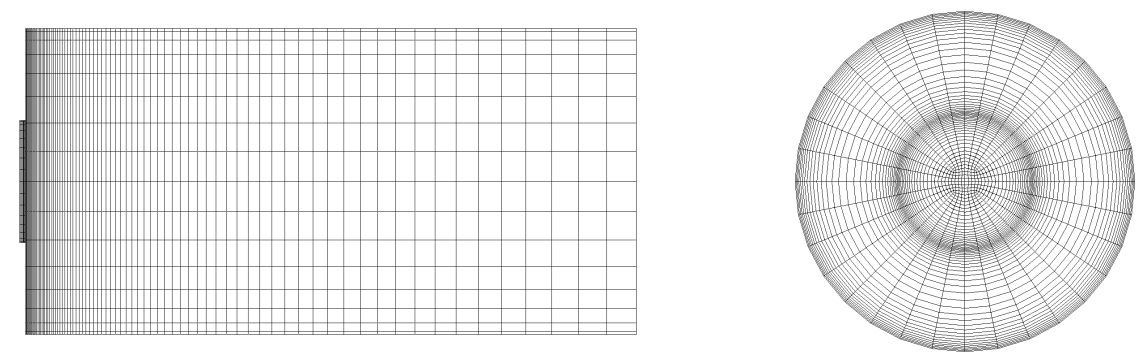

Figure 6: Two views of the 141,568 element expansion tube.

element expansion tube computational mesh are shown in Figure 6.

\subsubsection{Adjoint Sensitivity Analysis for turbulent SARANS CFD: 3D Turbulent Swirling Flow in Circular Expanding Pipe}

This example considers error estimation and adjoint sensitivity analysis for the swirling flow in the axisymmetric sudden expansion tube. The Reynolds number and swirl number were $R e=10,000$ and $S=0.04$ respectively. The Spalart-Allmaras turbulence model was used in these simulations. At this $R e$ and $S$, a steady-state solution is obtained [39]. The forward and adjoint sensitivity solution was obtained using Q1 elements. The mesh contained 300,000 elements and the axis of symmetry was along the z-axis. The baseline forward steady-state solution was obtained by pseudo-transient to steady-state time marching, requiring 180 time steps. The maximum time step reached the user-supplied limit $\Delta t_{\max }=10$ which is equivalent to a Courant-Friedrichs-Lewy number (CFL) equal to $10^{5}$, and the solution was integrated to 250 time units. Large CFL numbers are possible as time marching approaches steady-state. It is advantageous to use such large time steps in order to reduce overall time to solution. Following this psuedo-transient solution, a direct-to-steady-state solution was computed from this initial guess in three Newton steps in order to eliminate all fluctuations from the solution. Parameter sensitivities were computed by using the forward solution in the definition of the adjoint problem and carrying out a low-order adjoint solve for the adjoint derivatives as described in Section 3.4. To demonstrate the accuracy of the computed adjoint sensitivities, a study that is described below compares the prediction of a local linear Taylor series model using the 
computed adjoint derivatives to that of direct evaluation of the QoI by Drekar::CFD at a nearby parameter value. In this study forward solutions for the eight perturbed solutions $(\mu \pm 1 \%, \pm 10 \%)$ and $\left(G_{0} \pm 1 \%, \pm 10 \%\right)$ were achieved by solving direct-to-steady-state using the baseline/nominal solution as an initial guess. These direct-to-steady-state solutions required two Newton steps for the $1 \%$ perturbation and three Newton steps for the $10 \%$ perturbation.

In Figure 7 the x-component of velocity contours on a surface of constant z-component of velocity is presented which represent the rotating fluid core along with streamlines that show the complex recirculating flow patterns. In this case the $R e$ was the same however, swirl number was $(S=0.08)$. The parameters of interest in these initial numerical sensitivity studies are the dynamic viscosity $(\mu)$ and the pressure gradient $\left(G_{0}\right)$. Two QoI were computed. The first QoI(1) was the average turbulent viscosity in the entire domain:

$$
\overline{\hat{\nu}}=\int_{\Omega} \hat{\nu} d \Omega
$$

and the second QoI(2) was the average cross-stream kinetic energy:

$$
K E_{C R}=\int_{\Omega} \frac{1}{2} \rho\left(u_{x}^{2}+u_{y}^{2}\right) d \Omega .
$$

The local linear Taylor series model that was used to demonstrate the accuracy of the adjoint derivatives, and could optionally be used as a local surrogate model for sampling UQ, was constructed as;

$$
q=\bar{q}+\frac{\overline{\partial q}}{\partial p}(p-\bar{p})+\text { H.O.T. }
$$

where H.O.T. are higher order terms neglected by this linear model. Table 9 presents

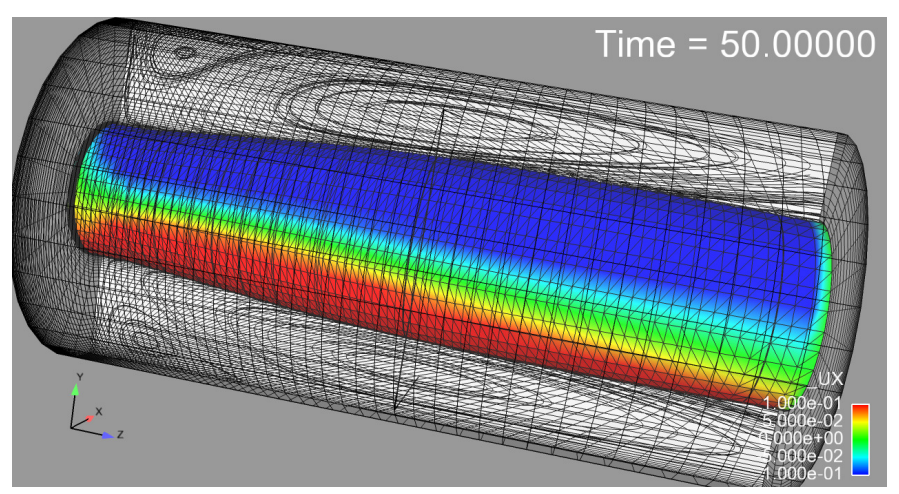

Figure 7: Image of axisymmetric sudden expansion tube at steady-state conditions with $S=0.08$. Iso-surface of z-component of velocity colored by x-component of velocity, taken from [33].

the computed values of the two QoI and the derivatives of the two QoI w.r.t. dynamic viscosity obtained by solving the adjoint problem. Table 10 shows a comparison of evaluating the linear surrogate for the QoI obtained from the adjoint derivative to the value computed in Drekar::CFD, in this case for a variation in the viscosity, $\mu$. For small local 


\begin{tabular}{|c|c|c|}
\hline QoI & QoI computed from Drekar::CFD & Adjoint Sensitivity \\
\hline \hline$\hat{\nu}$ & $3.866 \mathrm{e}+1$ & $-3.742 \mathrm{e}+6$ \\
\hline$K E_{C R}$ & $1.004 \mathrm{e}-2$ & -248.27 \\
\hline
\end{tabular}

Table 9: Estimate of two QoI and adjoint derivatives for swirling flow in axisymmetric sudden expansion tube at $R e=10,000$ and $S=0.08$.

\begin{tabular}{|c|c|c|c|c|c|c|}
\hline $\begin{array}{c}\text { Dynamic } \\
\text { Viscosity }\end{array}$ & $\begin{array}{c}\hat{\nu} \text { Comp. } \\
\text { Drekar::CFD }\end{array}$ & $\begin{array}{c}\hat{\nu} \text { Est. } \\
\text { from Adjoint }\end{array}$ & $\begin{array}{c}\text { Rel. } \\
\text { Error }\end{array}$ & $\begin{array}{c}K E_{C R} \text { Comp. } \\
\text { Drekar::CFD }\end{array}$ & $\begin{array}{c}K E_{C R} \text { Est. } \\
\text { Adjoint }\end{array}$ & $\begin{array}{c}\text { Rel. } \\
\text { Error }\end{array}$ \\
\hline \hline $1.01 \mathrm{e}-5$ & $3.82937 \mathrm{e}+1$ & $3.82899 \mathrm{e}+1$ & $0.01 \%$ & $1.00122 \mathrm{e}-2$ & $1.00125 \mathrm{e}-2$ & $0.003 \%$ \\
\hline $0.99 \mathrm{e}-5$ & $3.90421 \mathrm{e}+1$ & $3.90383 \mathrm{e}+1$ & $0.01 \%$ & $1.00620 \mathrm{e}-2$ & $1.00622 \mathrm{e}-2$ & $0.002 \%$ \\
\hline $1.10 \mathrm{e}-5$ & $3.52712 \mathrm{e}+1$ & $3.49222 \mathrm{e}+1$ & $1.0 \%$ & $9.74522 \mathrm{e}-3$ & $9.78914 \mathrm{e}-3$ & $0.450 \%$ \\
\hline $0.9 \mathrm{e}-5$ & $4.28242 \mathrm{e}+1$ & $4.24060 \mathrm{e}+1$ & $0.97 \%$ & $1.02835 \mathrm{e}-2$ & $1.02856 \mathrm{e}-2$ & $0.020 \%$ \\
\hline
\end{tabular}

Table 10: Adjoint-based Derivative used for Linear Taylor Series Model Estimate for the Swirling Expanding Pipe Problem.

variations in parameter space, these results suggest that a surrogate model constructed using Taylor's series and the adjoint derivative could be used instead of repeated forward solutions of the perturbed parameter which will greatly reduce the computational cost of the UQ. Similar results were obtained when considering the variation of the two QoI with the pressure gradient $G_{0}$ however the results are not presented. Clearly, while the local linear surrogate model has been demonstrated to have utility, this type of model is of limited use over a broader variation of a complex parameter space. In Section 5.2.3 however, we demonstrate that the use of the adjoint gradients in addition to the 3D turbulent flow function evaluations does enable the construction of more accurate surrogate models. Finally, in Figure 8 a sensitivity bar graph is presented that uses derivatives computed for the cross-stream KE QoI w.r.t. to the density, viscosity, inlet swirl and the inlet axial velocity ${ }^{1}$. This local sensitivity measure is defined by the following manipulation of the linear Taylor series model:

$$
\% \text { change in } \mathrm{q}=\left[\frac{q-\bar{q}}{\bar{q}}\right]_{p_{i}} * 10^{2} \approx \frac{\bar{p}_{i}}{\bar{q}} \frac{\overline{\partial q}}{\partial p_{i}}
$$

and provides a measure of the sensitivity of a specific QoI to the variation of the parameters in the simulation. That is for a variation of $1 \%$ in a parameter, this sensitivity measures locally the percentage change expected in the QoI if the true variation reflects a linear model locally. In the case considered here the inlet boundary condition swirl has the largest impact on the QoI. In this figure no attempt has been made to aggregate the parameters of interest into a more limited set of non-dimensional parameters (for example the Reynolds number). In Section 5.2.2 we consider a turbulent heat transfer

\footnotetext{
${ }^{1}$ It should be pointed out that at the time of submission of the manuscript the computation of the derivative of the QoI w.r.t. boundary data is carried out by a 5 pt finite difference approximation. We are currently carrying out an implementation in terms of adjoint derivatives.
} 
problem and present the sensitivities of multiple quantities of interest to both the naturally occurring physical parameters as well as the aggregated governing non-dimensional parameters of interest. Finally we point out that to obtain comparable local sensitivity information for $N_{P}$ uncertain parameters by using only function evaluation would require approximately $N_{p}$ more $3 \mathrm{D}$ turbulent flow solutions for a first-order finite difference approximation, or approximately $2 N_{P}$ for a second order centered approximation.

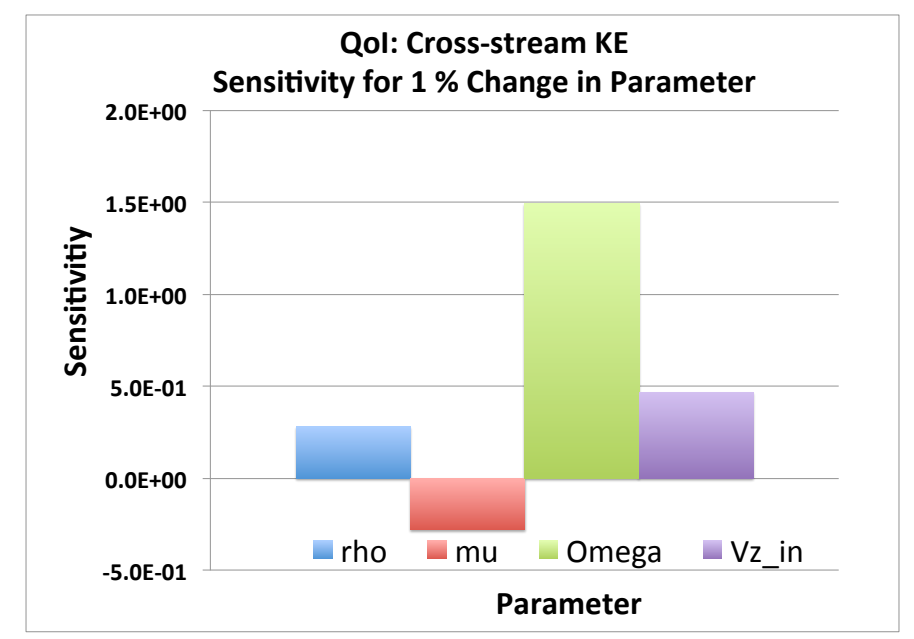

Figure 8: $1 \%$ Sensitivity for cross-stream KE QoI in the fully developed swirling flow for $R e=10,000$ and $S=0.1$.

\subsubsection{Adjoint-based Error Estimation Turbulent Swirling Flow in Expanding Pipe}

In this section we consider the use of adjoint-based error estimation in the same axisymmetric swirling sudden expansion flow. The Reynolds number and turbulence model are the same as presented in the previous section and the QoI is taken as the cross-stream KE. The result here that is of interest, is evaluating the use of the adjointbased error-estimation methods outlined in Section 3.4 to provide some understanding or measure of the error that is expected in specific QoI for a given spatial discretization of the full 3D turbulence computation.

The results for an initial study that is carried out for three different discretizations of the geometry and for two swirl numbers is presented in Table 11 and Table 12. The three different discretizations subdivide the angular discretization by a factor of 2 for each refinement and then also refine and adjust the nonuniform $x, y$ discretization to obtain a higher resolution. In each table the evaluation of the QoI, the adjoint error-estimate and a computation of the relative error and effectivity ratio is presented. In this context the final $8 M$ unknown discretization is taken as a reference. The data indicates that for the coarsest problem the adjoint error-estimate is off by approximately $2 \mathrm{x}$, which is in this case an upper bound. Upon one more refinement a very reasonable estimate of the error in the solution is obtained for a challenging 3D turbulent flow simulation. 


\begin{tabular}{|c|c|c|c|c|c|c|}
\hline $\begin{array}{c}\text { No. } \\
\text { Unknowns }\end{array}$ & QoI & $\begin{array}{c}\text { Error } \\
\text { Estimate }\end{array}$ & Prediction & $\begin{array}{c}\text { Rel. Err. } \\
\text { Adj. Est. }\end{array}$ & $\begin{array}{c}\text { Rel. Err. } \\
\text { Ref. Est. }\end{array}$ & $\begin{array}{c}\text { Eff. Rel. } \\
\text { Ref. }\end{array}$ \\
\hline \hline $288 \mathrm{k}$ & $5.63 \mathrm{E}-03$ & $5.86 \mathrm{E}-03$ & $1.15 \mathrm{E}-02$ & $104 \%$ & $57.5 \%$ & 1.81 \\
\hline $1.2 \mathrm{M}$ & $1.12 \mathrm{E}-02$ & $1.78 \mathrm{E}-03$ & $1.30 \mathrm{E}-02$ & $15.9 \%$ & $15.6 \%$ & 1.02 \\
\hline $8 \mathrm{M}$ & $1.33 \mathrm{E}-02$ & & & N.A. & N.A. & N.A. \\
\hline
\end{tabular}

Table 11: Error estimation for Swirl flow with $\operatorname{Re}_{D}=10 e 4$ and $S=0.1, \Omega=25$.

\begin{tabular}{|c|c|c|c|c|c|c|}
\hline $\begin{array}{c}\text { No. } \\
\text { Unknowns }\end{array}$ & QoI & $\begin{array}{c}\text { Error } \\
\text { Estimate }\end{array}$ & Prediction & $\begin{array}{c}\text { Rel. Err. } \\
\text { Adj. Est. }\end{array}$ & $\begin{array}{c}\text { Rel. Err. } \\
\text { Ref. Est. }\end{array}$ & $\begin{array}{c}\text { Eff. Rel. } \\
\text { Ref. }\end{array}$ \\
\hline \hline $288 \mathrm{k}$ & $1.15 \mathrm{E}-04$ & $1.51 \mathrm{E}-04$ & $2.66 \mathrm{E}-04$ & $131 \%$ & $71.1 \%$ & 1.84 \\
\hline $1.2 \mathrm{M}$ & $3.48 \mathrm{E}-04$ & $4.93 \mathrm{E}-05$ & $3.97 \mathrm{E}-04$ & $14.2 \%$ & $12.9 \%$ & 1.10 \\
\hline $8 \mathrm{M}$ & $3.99 \mathrm{E}-04$ & & & N.A. & N.A. & N.A. \\
\hline
\end{tabular}

Table 12: Error estimation for Swirl flow with $R e_{D}=10 e 4$ and $S=0, \Omega=0$.

\subsection{Fully Developed Turbulent Pipe Flow}

In this final study, the fully developed pipe flow problem is revisited. In this case the Reynolds numbers are high enough such that the flow is fully developed and turbulent. Simulations were conducted using the SARANS turbulence model. First, evidence that the steady-state solutions are accurate given adequate near wall spacing is presented. Next, sensitivities for three QoI and six parameters are are computed. Finally, surrogate models are constructed and a UQ study is performed.

\subsubsection{Verification of Turbulent Pipe Flow}

Consider heat transfer from the wall in fully developed turbulent pipe flow. This situation is again realized by applying periodic inflow-outflow boundary conditions and a momentum source in the axial direction.

The accuracy of the RANS turbulence model is assessed by comparing the computed universal law-of-the-wall to the theoretical result. The non-dimensional wall normal distance $y^{+}$and velocity $U^{+}$are determined from the wall shear $\tau_{w}$ and kinematic viscosity $\nu$

$$
y^{+}=u_{\tau} y / \nu \quad u_{\tau}=\sqrt{\frac{\tau_{w}}{\rho}} \quad U^{+}=\frac{u(y)}{u_{\tau}} .
$$

The law-of-the-wall relates $y^{+}$and $U^{+}$in the inner sub-layer next to the wall and the outer fully developed log-layer of a turbulent boundary layer;

$$
\begin{aligned}
& U^{+}=y^{+}(\text {viscous sub layer }) \\
& \left.U^{+}=\frac{1}{\kappa} \ln \left(y^{+}\right)+5.2 \quad \text { (log layer }\right) .
\end{aligned}
$$

and $\kappa$ is the von Karman constant take to be 0.41. Figures 9 show the mean radial velocity profiles normalized by the center-line velocity and a comparison of computed 
and theoretical law-of-the-wall respectively. Table 13 lists the computational details for this comparison. The solutions were achieved by pseudo-transient to steady-state time marching. These results show that given adequate near wall resolution $\left(y^{+} \leq \approx 10\right)$ the non-dimensional velocity profile (law-of-the-wall) is accurately reproduced.

\begin{tabular}{|c|c|c|c|c|c|}
\hline \#Elem & Model & CFL(max) & Time (sec.) & Reynolds no. & $y^{+}$ \\
\hline 52,400 & SA & 9,048 & 20 & 307,800 & 11.75 \\
\hline 62,000 & SA & 1.88 & 20 & 307,800 & 7.71 \\
\hline 78,000 & SA & 42,760 & 20 & 307,800 & 1.18 \\
\hline 86,000 & SA & 44,467 & 20 & 307,800 & 0.55 \\
\hline
\end{tabular}

Table 13: Summary of 3D Turbulent Pipe runs.
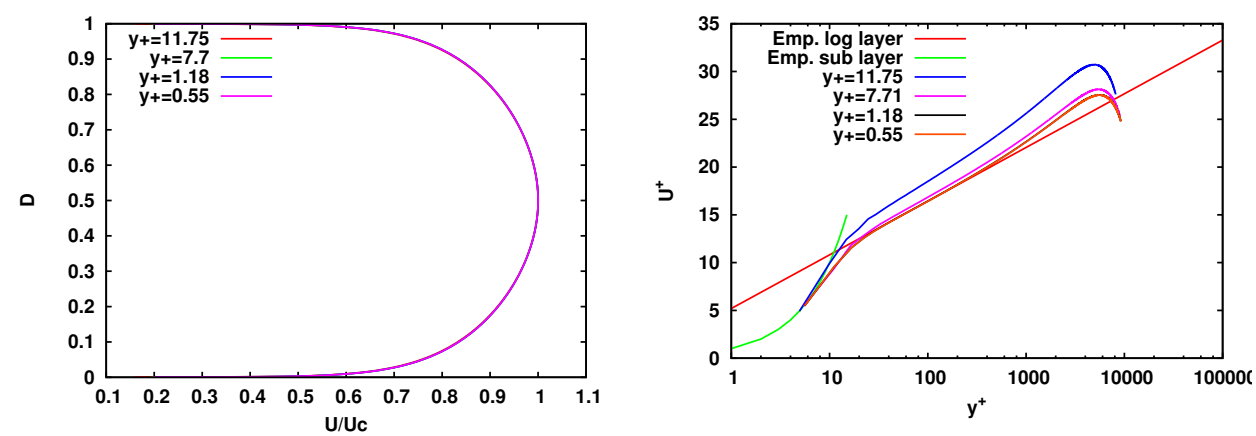

Figure 9: Mean velocity profiles for the tube with the Spalart-Allmaras turbulence model and law-ofthe-wall.

For the case of fully developed turbulent pipe flow, the time averaged velocity profile can be approximated by a one-seventh power law. For $\operatorname{Pr}$ near unity, Reynold's analogy states that the temperature profile will be similar to the velocity profile [22], thus,

$$
\frac{\bar{u}}{\bar{u}_{c}}=\left(1-\frac{r}{r_{w}}\right)^{1 / 7} \quad \frac{\bar{T}-T_{w}}{\bar{T}_{c}-T_{w}}=\left(1-\frac{r}{r_{w}}\right)^{1 / 7},
$$

where $\bar{u}_{c}$ and $\bar{T}_{c}$ are centerline values to velocity and temperature respectively. Using this assumption and the assumption that $P r_{t} \approx 1.0$, Kays\&Crawford [22] derived Nusselt numbers based on the mean velocity and temperature profiles that are a functions of $\mathrm{Pr}$ and $\operatorname{Re}_{D}$ ([22] eq. 13-8);

$$
N u=\frac{0.152 \operatorname{Re} e_{D}^{0.9} \operatorname{Pr}}{0.833\left[2.25 \ln \left(0.114 \operatorname{Re}_{D}^{0.9}\right)+13.2 \operatorname{Pr}-5.8\right]}
$$

and for lower $\operatorname{Pr}$ in the range [0.5-1.0], a simpler expression was derived ( [22] eq. 13-9);

$$
N u=0.022 \operatorname{Pr}^{0.5} \operatorname{Re}_{D}^{0.8} \quad \operatorname{Re}_{D}<10^{5} .
$$

The thermal entry length for turbulent flow is much longer than the laminar case. It is estimated from Kays\&Crawford that for $\frac{x}{D}=20$, the length of the computational domain, the error in $N u$ is approximately $2 \%$ ([22] p. 264). 
Nusselt numbers were computed to compare with these two expressions. Similar to the laminar case discussed in section 4.1.1, the temperature equation was solved independent of the flow, assuming a constant inflow value and the steady-state fully developed flow. The computational mesh contained 167,936 elements. The Reynolds number varied from 20,160-95,660, $\operatorname{Pr}$ varied from 0.5-1.5 and $P r_{t}$ varied from 0.5-2.0. A summary of the flow conditions is shown in Table 14. The Nusselt number comparisons are presented

\begin{tabular}{|c|c|c|c|c|}
\hline$R e_{D}$ & $y^{+}$ & $\Delta y_{\min }$ & $U_{z} \max$. & $U_{z}$ ave. \\
\hline \hline 20,160 & 1.69 & $1.513 \mathrm{e}-5$ & 2.48 & 2.016 \\
\hline 44,150 & 3.39 & $1.513 \mathrm{e}-5$ & 5.33 & 4.415 \\
\hline 95,660 & 6.71 & $1.513 \mathrm{e}-5$ & 11.39 & 9.566 \\
\hline
\end{tabular}

Table 14: Fully developed turbulent pipe flow with The Spalart-Allmaras model.

in Figure 10. In the left panel, $\operatorname{Pr}_{t}=1.0$ is held constant and $\operatorname{Pr}$ is allowed to vary. Numerical predictions show the correct slopes consistent with the correlations for low $\mathrm{Pr}$, but for higher $P r$, the numerical result lies in between the two correlations. In the right panel, $\operatorname{Pr}=1$ is held constant, and $\operatorname{Pr}_{t}$ varies. The computations show that consistent with the assumption inherent in Reynolds analogy $\left(P r_{t}=1.0\right)$, the predictions are in agreement with the correlations.
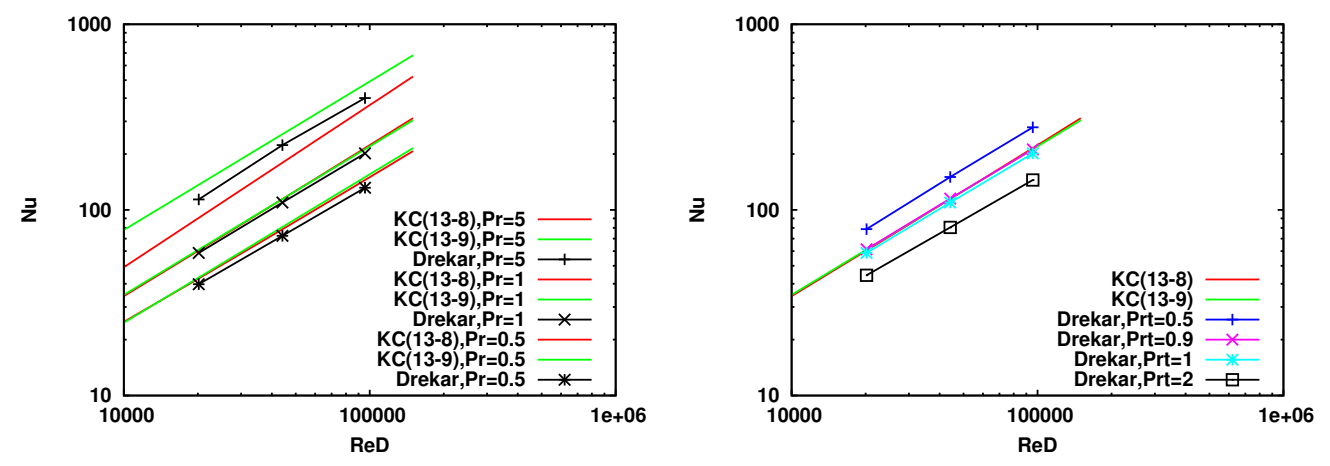

Figure 10: Comparisons of $N u$ vs. $\operatorname{Re}_{D}$ between Kays\&Crawford [22] (eq. 13-8) and Drekar::CFD for constant heat flux wall BCs (left) $\operatorname{Pr}=0.5,1.0,1.5, P r_{t}=1.0$ and (right) $\operatorname{Pr}=1.0, P r_{t}=$ $0.5,0.9,1.0,2.0$.

\subsubsection{Sensitivity of QoI in Fully Developed Turbulent Pipe Flow}

In this brief section we consider the results on a local sensitivity study for the heat transfer problem in a cylindrical pipe for a fully developed turbulent fluid flow approximated with the SARANS model. In Figure 11, the results of a local sensitivity study for six parameters is presented. The QoI in this case are the tube wall temperature, $T_{w}$, the mixing cup temperature, $T_{m}$ and $N u$ at the exit of the cylinder pipe. Briefly a few observations are described. With respect to mixing cup temperature $T_{m}$, it is clear that the variation w.r.t. to thermal conductivity and turbulent Prandtl number is 
effectively zero for this constant heat flux case. This is clearly understandable from the definition of $T_{m}$ and the fact that for a constant heat flux boundary the thermal energy must be absorbed (in steady state) by the exiting fluid. These particular parameters would change the gradients in the temperature profile but not the integrated QoI, $T_{m}$. Conversely, for the wall temperature it is clear that all the variables influence this QoI. For example to transfer the constant energy flux, for lower thermal conductivity, $k$, a higher wall temperature would be the result.

Finally in image (c) of Figure 11 a sensitivity plot for the variation of $N u$ is presented with the aggregated non-dimensional parameters of the $R e_{D}$ and $\operatorname{Pr}$ along with the SARANS turbulent heat transfer model parameter for the $P r_{t}$. From this plot it is clear that the $R e_{D}$ has the most influence on $N u$ at these (nominal conditions) and that the $\mathrm{Pr}_{t}$ also has a significant role in influencing $N u$. These sensitivities can be qualitatively validated by considering the Kays\&Crawford correlation (Equation 43) and taking derivatives of this equation and forming the sensitivities for the given parameters of this study. From the figure we see a very close agreement with the local sensitivity analysis of Drekar::CFD indicating the usefulness of these types of results.

\subsubsection{Quantification of the Uncertainty in the QoI in Fully Developed Pipe Flow}

In this section, we assume that the parameters studied in the previous section are uniformly distributed over user-defined ranges and assess the impact of this uncertainty on the quantity of interest. These parameter ranges for the six dimensional parameter space are given by:

$$
\begin{gathered}
\rho \in[0.5,2], \quad \mu \in[5 E-7,5 E-6], \quad-d p / d z \in[2.5,7.5], \\
C_{p} \in[0.1,10], \quad \kappa \in[5 E-7,5 E-6], \quad \operatorname{Pr}_{t} \in[0.1,10] .
\end{gathered}
$$

Since realistically we are limited in most cases in the number of model evaluations that we can perform, we use a small number of model evaluations to construct a surrogate model, also referred to as a response surface approximation. Then from the surrogate, that can be evaluated very quickly, we can use a large number of samples to estimate the statistics on the QoI. This approach has become quite common in recent years and a wide variety of surrogate models exist $[1,45,15,2]$. In this study, we choose to employ a Gaussian Process (GP) model to emulate the response surface. GP models produce smooth surrogate models of the response values from a set of data points. The smoothness of the approximation will depend on the correlation function that is used. In this study, we assume a Gaussian correlation function. We use DAKOTA[1] to construct the GP model, to perform the subsequent sampling of the surrogate model, and use these samples to estimate the statistics of the QoI. Naturally, the accuracy of these statistics depend on the accuracy of each sample of the surrogate model. Therefore, we are interested in using derivative information, provided by the adjoint solve, to improve the accuracy of the GP approximation. We compute the derivative information by solving the appropriate adjoint problem and pass this information to DAKOTA, which uses this additional information to construct the GP model as described in [1].

This study considers the wall temperature at the exit of the pipe, $T_{w}$, as the QoI. We use a Latin hypercube strategy to produce 80 samples over the range of the input parameters and use Drekar::CFD to compute the QoI and the gradient of the QoI at these sample points. Each of these function evaluations is a direct-to-steady-state solve 


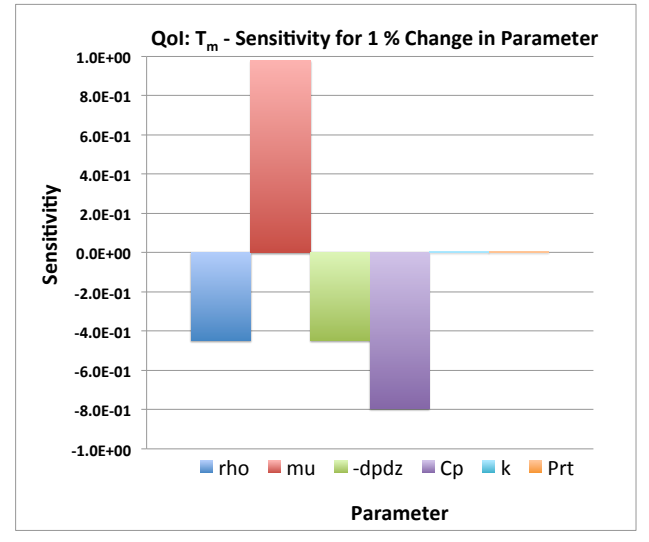

(a) QoI: $T_{m}$

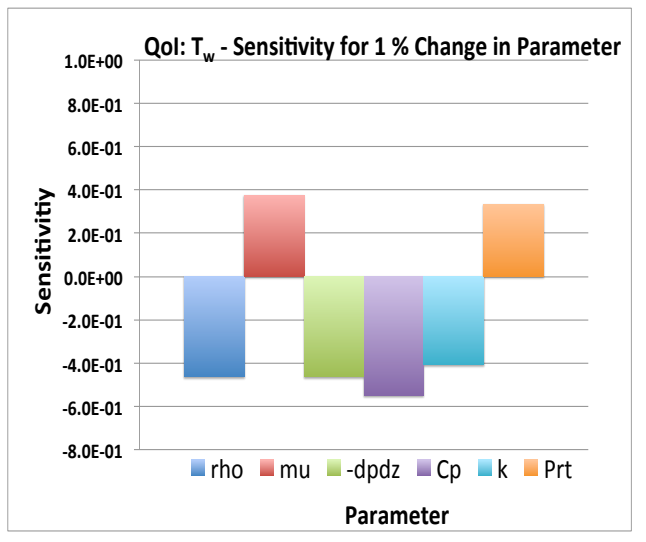

(b) QoI: $T_{w}$

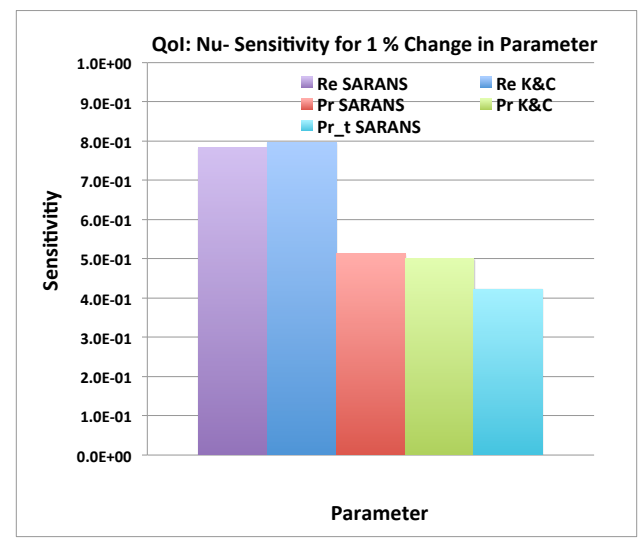

(c) QoI: $N u$

Figure 11: 1\% Sensitivity for QoI in the fully developed pipe flow to mixing temperature and wall temperature.

of the 3D coupled SARANS turbulent fluid flow and heat transfer problem of the $168 \mathrm{~K}$ element mesh with 1M unknowns. Additionally we solve the low-order adjoint problem to compute the six derivatives at each sample point. We use these model evaluations and gradients to construct a GP approximation and a gradient-enhanced GP approximation. We then use a separate Latin hypercube strategy to generate 68 additional samples over the range of parameters. We use Drekar::CFD to compute the QoI at these points. This second set of model evaluations is used to cross-validate the accuracy of the surrogate approximations. The average relative error at these cross-validation points is $29 \%$ for the GP model and 10\% for the gradient-enhanced GP model. Clearly, the extra information provided by the gradient significantly improves the accuracy of the surrogate model.

Next, we compare the statistics of the QoI using 100,000 samples of each of the surrogate models. The approximations of the mean are 20.11 and 20.79 for the GP model 
and the gradient-enhanced GP model respectively. The approximations of the standard deviation are 15.62 and 15.58 for the GP model and the gradient-enhanced GP model respectively. The approximations of the cumulative distribution functions are given in Figure 12. These results show the rather steep Cumulative Distribution Function (CDF) and small probability of obtaining QoI for the pipe wall temperature above 70 in this six dimensional parameter space. Finally using samples from the adjoint-enhanced GP surrogate model the Sobol indices are computed and are presented in Figure 13. These indices provide information similar to the sensitivity results presented earlier, however these results provide a global indication of the proportion of the variance in the QoI that can be attributed to variations in each uncertain parameter. In this regard, it is clear that the variation of the QoI over this space is mostly due to the variation of the specific heat and molecular thermal conductivity in the assumed parameter ranges.

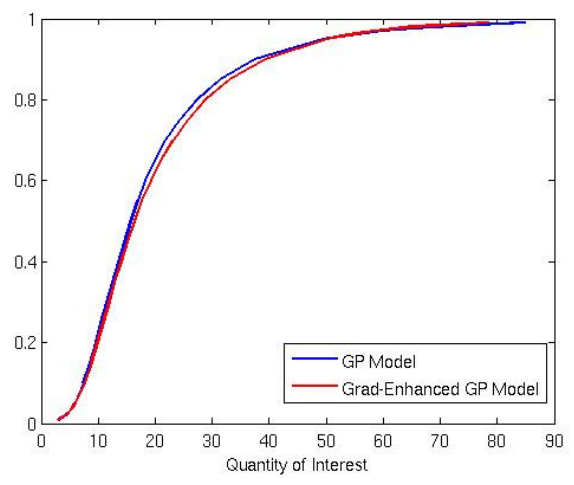

Figure 12: Cumulative distribution functions for the QoI using the GP model (blue) and the gradientenhanced GP model (red). 


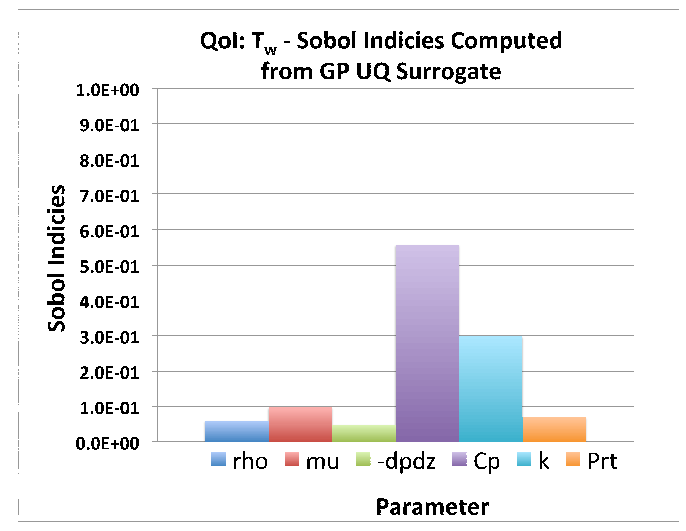

Figure 13: Sobol indices indicating the proportion of the variance in the quantity of interest that can be attributed to each uncertain parameter. These indices were computed using the gradient enhanced GP surrogate model. 


\section{Conclusions}

In this paper we have described a methodology for computing error estimates, performing sensitivity analysis and constructing surrogate models for uncertainty quantification. All of these beyond-forward simulation analysis capabilities make use of an adjoint solver embedded in the code architecture for which we have provided a high-level algorithmic overview. The adjoint solver makes use of much of the same code that is used for solving the forward problem, namely; data structures, Jacobian operator, residual operator and automatic differentiation. The code infrastructure is built on top of a fully implicit, fully coupled stabilized finite element method for solving nonlinear systems of PDEs.

Two separate investigations were conducted that showcase the power of this methodology. In the first investigation, high Reynolds number swirling turbulent flow through an axisymmetric sudden expansion tube was simulated by solving the RANS equations coupled to the Spalart-Allmaras turbulence model. Steady-state solutions were obtained. Next, two QoI were computed and their adjoint sensitivity. A comparison of the QoI computed directly and computed using a surrogate model showed that, at least, for small departures from the nominal values, the surrogate accurately represents the change in the QoI. This can be exploited in UQ studies where the surrogate substitutes for forward solutions. A comparison of the relative sensitivity to four parameters demonstrates the efficiency of this methodology. Additionally, an error-estimation study was presented where the adjoint-based error-estimate was compared to a finer mesh reference solution. The study indicated that for a modest resolution mesh, the adjoint-based computation for the relative error due to the numerical solution, was reasonably representative of the relative error directly computed by using the reference solution.

In the second investigation, fully developed turbulent pipe flow with heat transport was modeled. Correlations of the Nusselt number with Prandtl number and Reynolds number derived by Kays\&Crawford [22] were used to validate the solution to the forward problem. The local sensitivity of three QoI to six parameters was computed. These studies show the relative importance of different uncertain parameter to the heat transfer. This valuable information can be used to guide model development. For example, the sensitivity to the turbuent Prandtl number which is usually specified as a RANS model parameter can be assessed. Additionally, an example of developing a Gaussian Process response surface model for use in UQ studies was presented. Using this model Sobol indices were computed that assigned gobal variance contributions, over the six dimensional parameter space, to the various uncertain boundary condition, physical properties and model parameters.

Though these two investigations were for problems with relatively simple geometry and modest in size, this methodology can be utilized to address issues surrounding numerical discretization errors, sensitivities and UQ on more complex RANS multiphysics models in complex geometries. Based on results presented here, the tools/methodology described in this paper has the potential to greatly impact the assessment of computational models for application to CASL thermal-hydraulics problems. 


\section{Acknowledgements}

The authors thank Rick Garcia of Sandia National Laboratories for delivering $\mathrm{Cu}-$ bit based meshes of the $3 \times 3$ periodic rod/spacer grid reactor core sub-assembly model. Financial support from the CASL program is greatly appreciated. This work was also partially supported by the DOE Office of Science AMR program at Sandia National Laboratory under contract DE-AC04-94AL85000. 
[1] B.M. Adams, L.E. Bauman, W.J. Bohnhoff, K.R. Dalbey, M.S. Ebeida, J.P. Eddy, M.S. Eldred, P.D. Hough, K.T. Hu, J.D. Jakeman, L.P. Swiler, and D.M. Vigil. DAKOTA, A Multilevel Parallel Object-Oriented Framework for Design Optimization, Parameter Estimation, Uncertainty Quantification, and Sensitivity Analysis: Version 5.4 User's Manual. Technical Report SAND2010-2183, Sandia National Laboratories, 2009 (Updated 2013).

[2] I. Babuska, R. Tempone, and G. E. Zouraris. Galerkin finite element approximations of stochastic differential equations. SIAM J. Numer. Anal., 42(2):800-825, 2004.

[3] W. Bangerth and R. Rannacher. Adaptive Finite Element Methods for Differential Equations. Birkhauser Verlag, 2003.

[4] T. Barth, P. Bochev, M. Gunzburger, and J. Shadid. A taxonomy of consistently stabilized finite element methods for the Stokes problem. SIAM J. Sci. Comp., 25(5):1585-1607, 2004.

[5] R. Becker and R. Rannacher. An optimal control approach to a posteriori error estimation in finite element methods. Acta Numerica, 10:1-102, 2001.

[6] J. Blazek. Computational Fluid Dynamics: Principles and Applications. Elsevier Science LTD, Kidlington, Oxford, UK, 2001.

[7] A. N. Brooks and T.J.R. Hughes. Streamline upwind/Petrov-Galerkin formulations for convection dominated flows with particular emphasis on the incompressible Navier-stokes equations. Comp. Meth. Appl. Mech. and Eng., 32:199-259, 1982.

[8] D. G. Cacuci. Sensitivity and Uncertainty Analysis: Volume 1 Theory. Chapman and Hall/CRC, 2003.

[9] D. G. Cacuci, M. Ionescu-Bujor, and I.M. Navon. Sensitivity and Uncertainty Analysis: Volume 2 Applications to Large-Scale Systems. Chapman and Hall/CRC, 2005.

[10] E.C. Cyr, J.N. Shadid, and T.M. Wildey. Approaches for adjoint-based a posteriori analysis of stabilized finite element methods. SIAM Journal on Scientific Computing, 36:766-791, 2014.

[11] J. E. Dennis, Jr. and R. B. Schnabel. Numerical Methods for Unconstrained Optimization and Nonlinear Equations. Series in Automatic Computation. Prentice-Hall, Englewood Cliffs, NJ, 1983.

[12] J. Donea and A. Huerta. The Finite Element Method for Fluid Flow Problems. John Wiley and Sons, 2003

[13] S. C. Eisenstat and H. F. Walker. Choosing the forcing terms in an inexact Newton method. SIAM J. Sci. Comput., 17:16-32, 1996.

[14] K. Eriksson, D. Estep, P. Hansbo, and C. Johnson. Computational differential equations. Cambridge University Press, Cambridge, 1996. ISBN 0-521-56312-7; 0-521-56738-6.

[15] D. Estep and D. Neckels. Fast and reliable methods for determining the evolution of uncertain parameters in differential equations. J. Comput. Phys., 213:530-556, 2006.

[16] D. Estep, M. G. Larson, and R. D. Williams. Estimating the error of numerical solutions of systems of reaction-diffusion equations. Mem. Amer. Math. Soc., 146(696):viii+109, 2000. ISSN 0065-9266.

[17] M. Heroux et al. An overview of Trilinos. Technical Report Sand2003-2927, Sandia National Laboratories, Albuquerque NM, 87185, Aug. 2003.

[18] M. Gee, C. Siefert, J. Hu, R. Tuminaro, and M. Sala. ML 5.0 smoothed aggregation user's guide. Technical Report SAND2006-2649, Sandia National Laboratories, Albuquerque NM, 87185, 2006.

[19] L. Gilli, D. Lathouwers, J.L. Kloosterman, and T.H.J.J. van der Hagen. Application of sensitivity analysis to a simplified coupled neutronic thermal-hydraulics transient in a fast reactor using adjoint techniques. 2011. International Conference on Mathematical and Computational Methods Applied to Nuclear Science and Engineering.

[20] M. Gunzburger. Finite Element Methods for Viscous Incompressible Flows. Academic Press, Boston, 1989.

[21] T.J.R. Hughes, L. Franca, and M. Balestra. A new finite element formulation for computational fluid dynamics: V. Circumventing the Babuska-Brezzi condition: A stable Petrov-Galerkin formulation of the Stokes problem accommodating equal-order interpolations. Comput. Meth. Appl. Mech. Engrg., 59:85-99, 1986.

[22] W.M. Kays and M.E. Crawford. Convective Heat and Mass Transfer, 2nd Ed. McGraw-Hill Book Company, 1980.

[23] P. T. Lin, M. Sala, J. N. Shadid, and R. S. Tuminaro. Performance of fully-coupled algebraic multilevel domain decomposition preconditioners for incompressible flow and transport. Int. J. Num. Meth. Eng., 67:208-225, 2006.

[24] P. T. Lin, J. N. Shadid, R. S. Tuminaro, M. Sala, G. L. Hennigan, and R. P. Pawlowski. A parallel fully-coupled algebraic multilevel preconditioner applied to multiphysics PDE applications: Drift-diffusion, flow/transport/reaction, resistive MHD. Int. J. Num. Meth. Fluids, 64:1148-1179, 2010. 
[25] H. Mak and S. Balabani. Near field characteristics of swirling flow past a sudden expansion. Chemical Engineering Science, 62:6726-6746, 2007.

[26] P.K. Notz, R.P. Pawlowski, and J.C. Sutherland. Graph-based software design for managing complexity and enabling concurrency in multiphysics PDE software. ACM Transactions on Mathematical Software (TOMS), 39(1):1, 2012

[27] S. Pannala and A. Stagg. CASL THM: CFD benchmarks and test cases. Technical report, OakRidge National Laboratories, 2012.

[28] R.P. Pawlowski, E.T. Phipps, and A.G. Salinger. Automating embedded analysis capabilities and managing software complexity in multiphysics simulation, Part I: Template-based generic programming. Scientific Programming, 20(2):197-219, 2012.

[29] R.P. Pawlowski, E.T. Phipps, A.G. Salinger, S.J. Owen, C.M. Siefert, and M.L. Staten. Automating embedded analysis capabilities and managing software complexity in multiphysics simulation, Part II: Application to partial differential equations. Scientific Programming, 20(3):327-345, 2012.

[30] R.P. Pawlowski, J.N. Shadid, T.M. Smith, E. C. Cyr, and P.D. Weber. Drekar::CFD-a turbulent fluid-flow and conjugate heat transfer code: Theory manual version 1.0. Technical Report SAND2012-2697, Sandia National Laboratories, March 2012.

[31] A. Petruzzi. Development and Application of Methodologies for Sensitivity Analysis and Uncertainty Evaluation of the Results of the Best Estimate System Codes Applied in Nuclear Technology Sensitivities. Ph.D. dissertation, Universit di Pisa, December 2008.

[32] J. N. Shadid, A. G. Salinger, R. P. Pawlowski, P. T. Lin, G. L. Hennigan, R. S. Tuminaro, and R. B. Lehoucq. Stabilized FE computational analysis of nonlinear steady state transport/reaction systems. Comp. Meth. Applied Mech. Eng., 195:1846-1871, 2006.

[33] J. N. Shadid, T. M. Smith, R. P. Pawlowski, E. C. Cyr, and P.D. Weber. RANS CFD simulations for CASL THM using Drekar::CFD. Technical report, Sandia National Laboratories, October 31 2012 .

[34] J.N. Shadid, S.A. Hutchinson, G.L. Hennigan, H.K. Moffet, K.D. Devine, and A.G. Salinger. Efficient parallel computation of unstructured finite element reacting flow solutions. Parallel Computing, 23:1307-1325, 1997.

[35] J.N. Shadid, R.S. Tuminaro, K.D. Devine, G.L. Henningan, and P.T. Lin. Performance of fullycoupled domain decomposition preconditioners for finite element transport/reaction simulations. $J$. Comput. Phys., 205(1):24-47, 2005

[36] J.N. Shadid, R.P. Pawlowski, J.W. Banks, L. Chacon, P.T. Lin, and R.S. Tuminaro. Towards a scalable fully-implicit fully-coupled resistive MHD formulation with stabilized FE methods. Journal of Computational Physics, 229:7649-7671, 2010.

[37] J.N. Shadid, R.P. Pawlowski, E.C. Cyr, R.S. Tuminaro, L. Chacon, and P.D. Weber. Scalable implicit incompressible resistive MHD with stabilized FE and fully-coupled Newton-Krylov-AMG. Comp. Meth. Applied Mech. Eng., 304:1-25, 2016.

[38] F. Shakib. Finite element analysis of the compressible Euler and Navier-Stokes equations. PhD thesis, Division of Applied Mathematics, Stanford University, 1989.

[39] T.M. Smith, J. N. Shadid, R. P. Pawlowski, E. C. Cyr, T. M. Wildey, and P.D. Weber. Initial drekar RANS CFD simulations for CASL THM. Technical report, Sandia National Laboratories, February 2014

[40] P. R. Spalart and S. R. Allmaras. A One-Equation Turbulence Model for Aerodynamic Flows. AIAA Paper 92-0439, 1992.

[41] P.G. Tucker. Differential equation-based wall distance computation for DES and RANS. Journal of Computational Physics, 190:229-248, 2003.

[42] RS Tuminaro, CH Tong, JN Shadid, KD Devine, and DM Day. On a multilevel preconditioning module for unstructured mesh Krylov solvers: two-level Schwarz. Communications in numerical methods in engineering, 18(6):383-389, 2002.

[43] T.M. Wildey, E. C. Cyr, R.P. Pawlowski, J.N. Shadid, and T.M. Smith. Adjoint based a posteriori error estimation in Drekar::CFD. Technical Report SAND2012-8910, Sandia National Laboratories, October 2012.

[44] T.M. Wildey, E. C. Cyr, R.P. Pawlowski, J.N. Shadid, and T.M. Smith. A comparison of adjoint and data-centric verification techniques. Technical Report SAND2013-2879, Sandia National Laboratories, April 2013

[45] D. Xiu and G. Karniadakis. The Wiener-Askey polynomial chaos for stochastic differential equations. SIAM J. Sci. Comput., 24:619-644, 2002 